\title{
Aza-Prins-Pinacol Approach to 7-Azabicyclo[2.2.1]heptanes and Ring Expansion to [3.2.1]-Tropanes
}

\author{
Supporting Information
}

Alan Armstrong* and Stephen E. Shanahan

Imperial College, London 
$( \pm)-\left(1 R^{*}, 2 R^{*}, 4 S^{*}\right)$-2-Phenyl-7-(toluene-4-sulfonyl)-7-aza-bicyclo[2.2.1] heptane-2-carbaldehyde

$7 a$

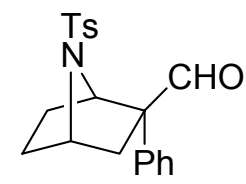

Magnesium turnings $(0.97 \mathrm{~g}, 40.0 \mathrm{mmol})$ were immersed with diethyl ether $(20 \mathrm{ml})$ in a reaction vessel equipped with reflux condenser. A solution of $\alpha$-bromo-styrene $(1.30 \mathrm{ml}$, $10.0 \mathrm{mmol})$ in diethyl ether $(3.7 \mathrm{ml})$ was added causing exothermic formation of the Grignard reagent. Separately, a solution of the aldehyde 5a $(1.73 \mathrm{~g}, 6.11 \mathrm{mmol})$ in diethyl ether $(30 \mathrm{ml})$ was cooled to $-30{ }^{\circ} \mathrm{C}$. The Grignard reagent solution $(18.3 \mathrm{ml}$, assumed concentration $0.40 \mathrm{M}, 7.34 \mathrm{mmol}$ ) was added via syringe, causing rapid precipitation. The reaction was allowed to warm to ambient temperature for $10 \mathrm{~min}$ before being quenched by addition of saturated aqueous $\mathrm{NH}_{4} \mathrm{Cl}$ solution $(50 \mathrm{ml})$. The mixture was extracted into diethyl ether $(2 \times 70 \mathrm{ml})$, the combined organics washed with brine and then dried over $\mathrm{Na}_{2} \mathrm{SO}_{4}$. Flash Column Chromatography (eluant $3: 2$ petroleum : diethyl ether) isolated the rearrangement precursor $\mathbf{6 a}$ as a mixture of diastereoisomers $(1.41 \mathrm{~g}$, $59 \%$ ) as a white foam; $v_{\max } / \mathrm{cm}^{-1} 3479,2987,2950,1598 ; m / z(\mathrm{CI}) 405\left[\mathrm{M}\left(\mathrm{NH}_{4}\right)^{+}\right.$, $10 \%$ ], $356\left[\left(\mathrm{MH}^{+}\right)-\mathrm{MeOH}, 100 \%\right.$; Found: $\mathrm{M}\left(\mathrm{NH}_{4}\right)^{+}$, 405.1838. $\mathrm{C}_{21} \mathrm{H}_{29} \mathrm{~N}_{2} \mathrm{O}_{4} \mathrm{~S}$ requires: 405.1848 .

To a $0{ }^{\circ} \mathrm{C}$ solution of rearrangement precursor $6 \mathbf{a}(50 \mathrm{mg}, 0.13 \mathrm{mmol})$ in dichloromethane $(1 \mathrm{ml})$ was added $\mathrm{SnCl}_{4}$ solution $(0.13 \mathrm{ml}, 1.0 \mathrm{M}$ in heptanes, 0.13 $\mathrm{mmol}$ ). The whole was stirred at $0{ }^{\circ} \mathrm{C}$ for $8 \mathrm{~min}$ before being quenched by addition of saturated aqueous $\mathrm{NaHCO}_{3}(5 \mathrm{ml})$. The mixture was extracted into dichloromethane $(2 \mathrm{x}$ $10 \mathrm{ml}$ ), dried over $\mathrm{Na}_{2} \mathrm{SO}_{4}$, filtered and solvent removed in vacuo to give aldehyde $7 \mathbf{a}$ (44 $\mathrm{mg}, 96 \%$ ) as a colourless oil; $v_{\max } / \mathrm{cm}^{-1}$ (film) 3061, 2985, 2961, 2881, 1727, 1598; $\delta_{\mathrm{H}}$ $\left(250 \mathrm{MHz}, \mathrm{CDCl}_{3}\right) 9.24(1 \mathrm{H}, \mathrm{s},-\mathrm{CHO}), 7.78\left(2 \mathrm{H}, \mathrm{d}, J 8.2,-\mathrm{SO}_{2}-\mathrm{C}-\mathrm{CH}\right), 7.41-7.27(5 \mathrm{H}$, m, Ar-H), 7.15-7.09 (2H, m, Ar-H), 4.82 (1H, d, J4.0, N-CH-C-CHO), 4.34-4.28 (1H, m, $\left.\mathrm{N}-\mathrm{CH}-\mathrm{CH}_{2}-\mathrm{C}-\mathrm{CHO}\right), 2.92$ (1H, ddd, J12.2, 5.2, 1.8, exo-CHH-C-CHO), 2.43 (3H, s, Ar$\left.\mathrm{CH}_{3}\right), 2.11-1.93$ (2H, m, -CHH-CHH- both exo), $1.75(1 \mathrm{H}, \mathrm{d}, J 12.2$, endo -CHH-C$\mathrm{CHO})$, 1.68-1.55 (1H, m, endo $\mathrm{CHH}-\mathrm{CH}(\mathrm{N})-\mathrm{C}-\mathrm{CHO}), 1.51-1.37$ (1H, m, endo $\mathrm{CHH}-$ $\left.\mathrm{CH}(\mathrm{N})-\mathrm{CH}_{2^{-}}\right) ; \delta_{\mathrm{C}}\left(125 \mathrm{MHz}, \mathrm{CDCl}_{3}\right) 197.0(\mathrm{CH}), 143.8(\mathrm{C}), 137.2(\mathrm{C}), 134.9(\mathrm{C}), 129.6$ $(\mathrm{CH}), 129.2(\mathrm{CH}), 128.6(\mathrm{CH}), 127.9(\mathrm{CH}), 127.7(\mathrm{CH}), 65.5(\mathrm{C}), 62.9(\mathrm{CH}), 59.6(\mathrm{CH})$, $37.0\left(\mathrm{CH}_{2}\right), 30.9\left(\mathrm{CH}_{2}\right), 23.9\left(\mathrm{CH}_{2}\right), 21.6\left(\mathrm{CH}_{3}\right) ; \mathrm{m} / z(\mathrm{CI}) 356\left(\mathrm{MH}^{+}, 60 \%\right)$; Found: $\mathrm{MH}^{+}$, 356.1315. $\mathrm{C}_{20} \mathrm{H}_{22} \mathrm{NO}_{3} \mathrm{~S}$ requires: 356.1320 . 
$( \pm)-\left(1 R^{*}, 3 R^{*}, 5 S^{*}\right)-3-P h e n y l-8$-(toluene-4-sulfonyl)-8-aza-bicyclo[3.2.1]octan-2-one 8a and $( \pm)-\left(1 R^{*}, 3 S^{*}, 5 S^{*}\right)$-3-Phenyl-8-(toluene-4-sulfonyl)-8-aza-bicyclo[3.2.1]octan-2-one 9a
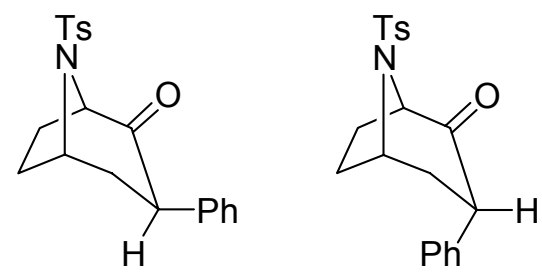

To a $0 \quad{ }^{\circ} \mathrm{C}$ solution of rearrangement precursor $\mathbf{6 a}(113 \mathrm{mg}, 0.29 \mathrm{mmol})$ in dichloromethane $(2.3 \mathrm{ml})$ was added $\mathrm{SnCl}_{4}$ solution $(0.58 \mathrm{ml}, 1.0 \mathrm{M}$ in heptanes, 0.58 $\mathrm{mmol})$. The mixture was allowed to warm to ambient temperature and stirred for $16 \mathrm{~h}$. The reaction was quenched by addition of saturated aqueous $\mathrm{NaHCO}_{3}(10 \mathrm{ml})$. The mixture was extracted into chloroform $(2 \times 20 \mathrm{ml})$ and combined organics dried over $\mathrm{Na}_{2} \mathrm{SO}_{4}$. Flash Column Chromatography (eluant $1: 1$ petroleum : diethyl ether) isolated the isomeric tropanes $\mathbf{8 a}$ (62 mg, $60 \%$, white crystals) and $9 \mathbf{a}(16 \mathrm{mg}, 16 \%$, white gum) as pure compounds; Data for 8a: m.p. $181-183{ }^{\circ} \mathrm{C} ; v_{\max } / \mathrm{cm}^{-1}$ (film) 3030, 2961, 2883, 1726,$1595 ; \delta_{\mathrm{H}}\left(250 \mathrm{MHz}, \mathrm{CDCl}_{3}\right) 7.81\left(2 \mathrm{H}, \mathrm{d}, J 8.1, \mathrm{SO}_{2}-\mathrm{C}-\mathrm{CH}\right), 7.34(2 \mathrm{H}, \mathrm{d}, J 8.1, \mathrm{Ar}-$ H), 7.31-7.24 (3H, m, Ar-H), 6.99-6.94 (2H, m, Ar-H), 4.63-4.56 [1H, m, N-CH- $\left.\left(\mathrm{CH}_{2}\right)_{2}\right]$, 4.41-4.36 (1H, m, N-CH-C=O), 3.59 (1H, dd, J11.6, 7.6, Ph-CH-C=O), 2.47 (3H, s, Ar$\left.\mathrm{CH}_{3}\right), 2.42-2.13\left(2 \mathrm{H}, \mathrm{m}, \mathrm{CH}_{2}-\mathrm{CH}-\mathrm{Ph}\right), 2.10-1.87\left(4 \mathrm{H}, \mathrm{m}, \mathrm{CH}_{2}-\mathrm{CH}_{2}\right) ; \delta_{\mathrm{C}}(125 \mathrm{MHz}$, $\left.\mathrm{CDCl}_{3}\right) 202.2(\mathrm{C}), 144.2(\mathrm{C}), 137.5(\mathrm{C}), 136.7(\mathrm{C}), 129.9(\mathrm{CH}), 129.0(\mathrm{CH}), 128.6(\mathrm{CH})$, $127.7(\mathrm{CH}), 127.3(\mathrm{CH}), 66.1(\mathrm{CH}), 57.1(\mathrm{CH}), 49.3(\mathrm{CH}), 41.6\left(\mathrm{CH}_{2}\right), 28.2\left(\mathrm{CH}_{2}\right), 27.6$ $\left(\mathrm{CH}_{2}\right), 21.6\left(\mathrm{CH}_{3}\right) ; \mathrm{m} / z(\mathrm{CI}) 373\left[\mathrm{M}\left(\mathrm{NH}_{4}\right)^{+}, 100 \%\right.$ ]; Found: $\mathrm{MH}^{+}, 356.1318 . \mathrm{C}_{20} \mathrm{H}_{22} \mathrm{NO}_{3} \mathrm{~S}$ requires: 356.1320 ;

Data for 9a: $v_{\max } / \mathrm{cm}^{-1}$ (film) 2957, 2929, 2873, 1730, 1597; $\delta_{\mathrm{H}}\left(250 \mathrm{MHz}, \mathrm{CDCl}_{3}\right) 7.79$ $\left(2 \mathrm{H}, \mathrm{d}, J 8.5, \mathrm{SO}_{2}-\mathrm{C}-\mathrm{CH}\right), 7.38-7.23$ (5H, m, Ar-H), 7.06-7.00 (2H, m, Ar-H), 4.66-4.48 (2H, m, CH-N-CH), 3.92 (1H, t, J11.3, Ph-CH-C=O), 3.01-2.86 (1H, m, CHH-CH-Ph), 2.46-2.37 (1H, m, CHH-CH-Ph), 1.87-1.38 (4H, m, CH $\left.\mathbf{C H}_{2}-\mathrm{CH}_{2}\right) ; \delta_{\mathrm{C}}\left(125 \mathrm{MHz}, \mathrm{CDCl}_{3}\right)$ 208.8 (C), $144.2(\mathrm{C}), 137.2(\mathrm{C}), 136.9(\mathrm{C}), 130.0(\mathrm{CH}), 128.9(\mathrm{CH}), 128.6(\mathrm{CH}), 127.3$ $(\mathrm{CH}), 125.7(\mathrm{CH}), 66.1(\mathrm{CH}), 55.3(\mathrm{CH}), 49.7(\mathrm{CH}), 36.9\left(\mathrm{CH}_{2}\right), 31.7\left(\mathrm{CH}_{2}\right), 28.3\left(\mathrm{CH}_{2}\right)$, $21.6\left(\mathrm{CH}_{3}\right) ; m / z(\mathrm{CI}) 373\left[\mathrm{M}\left(\mathrm{NH}_{4}\right)^{+}, 100 \%\right.$; Found: $\mathrm{M}\left(\mathrm{NH}_{4}\right)^{+}, 373.1582 . \mathrm{C}_{20} \mathrm{H}_{25} \mathrm{~N}_{2} \mathrm{O}_{3} \mathrm{~S}$ requires: 373.1586 . 
$( \pm)-\left(1 R^{*}, 2 R^{*}, 4 S^{*}\right)-2-M e t h y l-7-(t o l u e n e-4-s u l f o n y l)-7-a z a-b i c y c l o[2.2 .1]$ heptane-2-carbaldehyde $7 b$<smiles>CC1(C=O)C2CCC(C2)N1[TeH]</smiles>

Magnesium turnings $(0.58 \mathrm{~g}, 24.0 \mathrm{mmol})$ were immersed with diethyl ether $(20.5 \mathrm{ml})$ in a reaction vessel equipped with reflux condenser. A crystal of iodine was added and the mixture stirred until the iodine colour disappeared. An initial quantity of neat 2bromopropene $(0.20 \mathrm{ml})$ was added, initiating Grignard reagent formation. Further 2bromopropene $(0.33 \mathrm{ml})$ was added, maintaining reflux. Separately, a solution of the aldehyde 5a $(0.42 \mathrm{~g}, 1.48 \mathrm{mmol})$ in diethyl ether $(10 \mathrm{ml})$ was cooled to $-30{ }^{\circ} \mathrm{C}$. The Grignard reagent solution $(6.14 \mathrm{ml}$, assumed concentration $0.29 \mathrm{M}, 1.78 \mathrm{mmol})$ was added via syringe, causing rapid precipitation. The reaction was allowed to warm to ambient temperature for $10 \mathrm{~min}$ before being quenched by addition of saturated aqueous $\mathrm{NH}_{4} \mathrm{Cl}$ solution $(20 \mathrm{ml})$. The mixture was extracted into diethyl ether $(2 \times 20 \mathrm{ml})$, the combined organics washed with brine and then dried over $\mathrm{Na}_{2} \mathrm{SO}_{4}$. Flash Column Chromatography (eluant $3: 2$ petroleum : diethyl ether) isolated the rearrangement precursor $\mathbf{6 b}$ as a mixture of diastereoisomers $(0.19 \mathrm{~g}, 40 \%)$ as a colourless oil; $v_{\max } /$ $\mathrm{cm}^{-1}$ (film) 3492, 2979, 2951, 1651, 1598; m/z (CI) $343\left[\mathrm{M}\left(\mathrm{NH}_{4}\right)^{+}, 15 \%\right], 294\left[\left(\mathrm{MH}^{+}\right)-\right.$ $\mathrm{MeOH}, 100 \%$ ]; Found: $\mathrm{M}\left(\mathrm{NH}_{4}\right)^{+}, 343.1687 . \mathrm{C}_{16} \mathrm{H}_{27} \mathrm{~N}_{2} \mathrm{O}_{4} \mathrm{~S}$ requires: 343.1692 .

To a $0{ }^{\circ} \mathrm{C}$ solution of rearrangement precursor $\mathbf{6 b}(26 \mathrm{mg}, 0.08 \mathrm{mmol})$ in dichloromethane $(1 \mathrm{ml})$ was added $\mathrm{SnCl}_{4}$ solution $(80 \mu \mathrm{l}, 1.0 \mathrm{M}$ in heptanes, $0.08 \mathrm{mmol})$. The whole was stirred at $0{ }^{\circ} \mathrm{C}$ for $8 \mathrm{~min}$ before being quenched by addition of saturated aqueous $\mathrm{NaHCO}_{3}(5 \mathrm{ml})$. The mixture was extracted into dichloromethane $(2 \times 10 \mathrm{ml})$, dried over $\mathrm{Na}_{2} \mathrm{SO}_{4}$, filtered and solvent removed in vacuo to give aldehyde $7 \mathbf{b}$ (19 mg, 81 \%) as a colourless oil; $v_{\max } / \mathrm{cm}^{-1}$ (film) 2964, 2926, 1726, 1598; $\delta_{\mathrm{H}}\left(250 \mathrm{MHz}, \mathrm{CDCl}_{3}\right)$ $9.40(1 \mathrm{H}, \mathrm{s},-\mathrm{CHO}), 7.74\left(2 \mathrm{H}, \mathrm{d}, J 8.2, \mathrm{SO}_{2}-\mathrm{C}-\mathrm{CH}\right), 7.28(2 \mathrm{H}, \mathrm{d}, J 8.2, \mathrm{Ar}-\mathbf{H}), 4.21-4.16$ $(2 \mathrm{H}, \mathrm{m}, \mathrm{CH}-\mathrm{N}-\mathrm{CH}), 2.42\left(3 \mathrm{H}, \mathrm{s}, \mathrm{Ar}-\mathrm{CH}_{3}\right), 2.37$ (1H, ddd, J12.3, 5.5, 2.4, exo CHH$\mathrm{C}(\mathrm{Me})-\mathrm{C}=\mathrm{O}), 2.05-1.42\left(4 \mathrm{H}, \mathrm{m}, \mathrm{CH}_{2}-\mathrm{CH}_{2}\right), 1.08\left(3 \mathrm{H}, \mathrm{s}, \mathrm{CH}_{3}-\mathrm{C}-\mathrm{C}=\mathrm{O}\right), 1.04(1 \mathrm{H}, \mathrm{d}$, $J 12.3$, endo $\mathrm{CHH}-\mathrm{C}(\mathrm{Me})-\mathrm{C}=\mathrm{O}) ; \delta_{\mathrm{C}}\left(100 \mathrm{MHz}, \mathrm{CDCl}_{3}\right) 202.6(\mathrm{CH}), 143.8(\mathrm{C}), 137.1(\mathrm{C})$, $129.6(\mathrm{CH}), 127.6(\mathrm{CH}), 63.8(\mathrm{CH}), 60.1(\mathrm{CH}), 54.1(\mathrm{C}), 40.0\left(\mathrm{CH}_{2}\right), 30.3\left(\mathrm{CH}_{2}\right), 23.7$ $\left(\mathrm{CH}_{2}\right), 21.6\left(\mathrm{CH}_{3}\right), 16.8\left(\mathrm{CH}_{3}\right) ; \mathrm{m} / z(\mathrm{CI}) 311\left[\mathrm{M}\left(\mathrm{NH}_{4}\right)^{+}, 100 \%\right.$; Found: $\mathrm{M}\left(\mathrm{NH}_{4}\right)^{+}$, 311.1435. $\mathrm{C}_{15} \mathrm{H}_{23} \mathrm{~N}_{2} \mathrm{O}_{3} \mathrm{~S}$ requires: 311.1429 . 
$( \pm)-\left(1 R^{*}, 3 S^{*}, 5 S^{*}\right)-3-M e t h y l-8$-(toluene-4-sulfonyl)-8-aza-bicyclo[3.2.1]octan-2-one 8b and $( \pm)-\left(1 R^{*}, 3 R^{*}, 5 S^{*}\right)$-3-Methyl-8-(toluene-4-sulfonyl)-8-aza-bicyclo[3.2.1]octan-2-one 9b
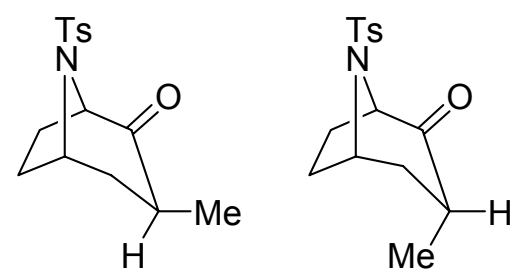

To a $0 \quad{ }^{\circ} \mathrm{C}$ solution of rearrangement precursor $\mathbf{6 b}(40 \mathrm{mg}, 0.12 \mathrm{mmol})$ in dichloromethane $(1 \mathrm{ml})$ was added $\mathrm{SnCl}_{4}$ solution $(0.24 \mathrm{ml}, 1.0 \mathrm{M}$ in heptanes, 0.24 mmol). The mixture was allowed to warm to ambient temperature and stirred for $16 \mathrm{~h}$. The reaction was quenched by addition of saturated aqueous $\mathrm{NaHCO}_{3}(10 \mathrm{ml})$. The mixture was extracted into chloroform $(2 \times 15 \mathrm{ml})$ and combined organics dried over $\mathrm{Na}_{2} \mathrm{SO}_{4}$. Flash Column Chromatography (eluant $1: 1$ petroleum : diethyl ether) isolated the isomeric tropanes $\mathbf{8 b}(13 \mathrm{mg}, 37 \%$, white gum) and $9 \mathbf{b}$ (6 mg, $17 \%$, colourless oil) as pure compounds; Data for $\mathbf{8 b}: v_{\max } / \mathrm{cm}^{-1}$ (film) 2961, 2924, 1724, 1595; $\delta_{\mathrm{H}}(250$ $\left.\mathrm{MHz}, \mathrm{CDCl}_{3}\right) 7.74\left(2 \mathrm{H}, \mathrm{d}, J 8.2, \mathrm{SO}_{2}-\mathrm{C}-\mathrm{CH}\right), 7.30(2 \mathrm{H}, \mathrm{d}, J 8.2, \mathrm{Ar}-\mathbf{H}), 4.48-4.40$ [1 H, m, $\left.\mathrm{N}-\mathrm{CH}-\left(\mathrm{CH}_{2}\right)_{2}\right], 4.29-4.23(1 \mathrm{H}, \mathrm{m}, \mathrm{N}-\mathrm{CH}-\mathrm{C}=\mathrm{O}), 2.51-2.34(1 \mathrm{H}, \mathrm{m}, \mathrm{CH}-\mathrm{Me}), 2.43(3 \mathrm{H}, \mathrm{s}$, Ar-CH $\left.\left.\mathrm{CH}_{3}\right), 2.03-1.66\left(6 \mathrm{H}, \mathrm{m}, \mathrm{C}-\mathrm{CH}_{2}-\mathrm{C}\right), 0.97(3 \mathrm{H}, \mathrm{d}, J 6.7, \mathrm{CH}-\mathrm{CH})_{3}\right) \delta_{\mathrm{C}}(100 \mathrm{MHz}$, $\left.\mathrm{CDCl}_{3}\right) 204.8(\mathrm{C}), 144.0(\mathrm{C}), 136.5(\mathrm{C}), 129.8(\mathrm{CH}), 127.6(\mathrm{CH}), 65.7(\mathrm{CH}), 57.1(\mathrm{CH})$, $40.8(\mathrm{CH}), 36.6\left(\mathrm{CH}_{2}\right), 27.8\left(\mathrm{CH}_{2}\right), 27.6\left(\mathrm{CH}_{2}\right), 21.6\left(\mathrm{CH}_{3}\right), 14.1\left(\mathrm{CH}_{3}\right) ; \mathrm{m} / z(\mathrm{CI}) 311$ $\left[\mathrm{M}\left(\mathrm{NH}_{4}\right)^{+}, 100 \%\right.$; Found: $\mathrm{M}\left(\mathrm{NH}_{4}\right)^{+}, 311.1426 . \mathrm{C}_{15} \mathrm{H}_{23} \mathrm{~N}_{2} \mathrm{O}_{3} \mathrm{~S}$ requires: 311.1429 ;

Data for 9b: $v_{\max } / \mathrm{cm}^{-1}$ (film) 2969, 2934, 2874, 1726, 1597; $\delta_{\mathrm{H}}\left(250 \mathrm{MHz}, \mathrm{CDCl}_{3}\right) 7.74$ $\left(2 \mathrm{H}, \mathrm{d}, J 8.1, \mathrm{SO}_{2}-\mathrm{C}-\mathrm{CH}\right), 7.30$ (2H, d, J8.1, Ar-H), 4.51-4.42 [1H, m, N-CH- $\left.\left(\mathrm{CH}_{2}\right)_{2}\right]$, 4.38-4.32 (1H, m, N-CH-C=O), 2.80-2.62 (1H, m, CH-CH $), 2.43\left(3 \mathrm{H}, \mathrm{s}, \mathrm{Ar}-\mathrm{CH}_{3}\right), 1.83-$ $0.86\left(6 \mathrm{H}, \mathrm{m}, \mathrm{C}-\mathrm{CH}_{2}-\mathrm{C}\right), 1.04\left(3 \mathrm{H}, \mathrm{d}, J 6.4, \mathrm{CH}-\mathrm{CH}_{3}\right) ; \delta_{\mathrm{C}}\left(125 \mathrm{MHz}, \mathrm{CDCl}_{3}\right) 211.6(\mathrm{C})$, $144.0(\mathrm{C}), 137.0(\mathrm{C}), 129.9(\mathrm{CH}), 127.3(\mathrm{CH}), 65.8(\mathrm{CH}), 55.1(\mathrm{CH}), 37.4(\mathrm{CH}), 36.3$ $\left(\mathrm{CH}_{2}\right), 31.5\left(\mathrm{CH}_{2}\right), 28.5\left(\mathrm{CH}_{2}\right), 21.5\left(\mathrm{CH}_{3}\right), 14.6\left(\mathrm{CH}_{3}\right) ; \mathrm{m} / z(\mathrm{CI}) 311\left[\mathrm{M}\left(\mathrm{NH}_{4}\right)^{+}, 100 \%\right]$; Found: 294.1158. $\mathrm{C}_{15} \mathrm{H}_{20} \mathrm{NO}_{3} \mathrm{~S}$ requires: 294.1164. 
$( \pm)-\left(1 R^{*}, 2 S^{*}, 3 R^{*}, 4 S^{*}\right)$-(trans-2,3-Dimethyl)-7-(toluene-4-sulfonyl)-7-aza-bicyclo[2.2.1]heptane2-carbaldehyde 7c<smiles>CC1C(O)C2CCC1C2(C)O</smiles>

A solution of (Z)-2-bromo-but-2-ene $(0.16 \mathrm{ml}, 1.55 \mathrm{mmol})$ in diethyl ether $(10 \mathrm{ml})$ was cooled to $-78{ }^{\circ} \mathrm{C}$. ${ }^{\mathrm{t}} \mathrm{BuLi}(1.82 \mathrm{ml}, 1.7 \mathrm{M}$ in pentane, $3.10 \mathrm{mmol})$ was added and the mixture stirred at $-78{ }^{\circ} \mathrm{C}$ for $1 \mathrm{~h}$. Solid $\mathrm{MgBr}_{2} \cdot \mathrm{OEt}_{2}(364 \mathrm{mg}, 1.41 \mathrm{mmol}$ ) was introduced and the temperature raised to $0{ }^{\circ} \mathrm{C}$ for $30 \mathrm{~min}$. Separately, the aldehyde $\mathbf{5 a}(367 \mathrm{mg}, 1.30$ mmol) was dissolved in diethyl ether $(7 \mathrm{ml})$ and the whole cooled to $0{ }^{\circ} \mathrm{C}$. The entire organometallic solution $\left(\right.$ at $0{ }^{\circ} \mathrm{C}$ ) was transferred into this via canula with stirring. Stirred a further 5 min at ambient temperature before quenching with saturated aqueous $\mathrm{NH}_{4} \mathrm{Cl}$ $(20 \mathrm{ml})$. The mixture was extracted into diethyl ether $(2 \times 20 \mathrm{ml})$ and combined organics dried over $\mathrm{Na}_{2} \mathrm{SO}_{4}$. Flash Column Chromatography (eluant $3: 2$ petroleum : diethyl ether, gradient rising to $1: 1$ ) isolated the desired rearrangement precursor $\mathbf{6 c}$ as a mixture of diastereoisomers $\left(177 \mathrm{mg}, 40 \%\right.$ ) as a colourless oil; $v_{\max } / \mathrm{cm}^{-1}$ (film) 3488 , 2978, 2946, 1598; $m / z(\mathrm{CI}) 357\left[\mathrm{M}\left(\mathrm{NH}_{4}\right)^{+}, 5 \%\right], 308$ [( $\left.\left.\mathrm{MH}^{+}\right)-\mathrm{MeOH}, 90 \%\right]$; Found: $357.1840\left[\mathrm{M}\left(\mathrm{NH}_{4}\right)^{+}\right] . \mathrm{C}_{17} \mathrm{H}_{29} \mathrm{~N}_{2} \mathrm{O}_{4} \mathrm{~S}$ requires: 357.1848 .

To a $0{ }^{\circ} \mathrm{C}$ solution of rearrangement precursor $6 \mathbf{c}(55 \mathrm{mg}, 0.16 \mathrm{mmol})$ in dichloromethane $(1 \mathrm{ml})$ was added $\mathrm{SnCl}_{4}$ solution $(0.16 \mathrm{ml}, 1.0 \mathrm{M}$ in heptanes, $0.16 \mathrm{mmol})$. The whole was stirred at $0{ }^{\circ} \mathrm{C}$ for $8 \mathrm{~min}$ before being quenched by addition of saturated aqueous $\mathrm{NaHCO}_{3}(5 \mathrm{ml})$. The mixture was extracted into dichloromethane $(2 \mathrm{x} 10 \mathrm{ml})$ and combined organics dried over $\mathrm{Na}_{2} \mathrm{SO}_{4}$. Flash Column Chromatography (eluant 2 : 1 petroleum : diethyl ether) afforded the aldehyde 7c (31 mg, 62\%) as white crystals, m.p. $129-130{ }^{\circ} \mathrm{C}(\mathrm{dec}) ; v_{\max } / \mathrm{cm}^{-1}$ (film) 2965, 2927, 2877, 1721; $\delta_{\mathrm{H}}\left(250 \mathrm{MHz}, \mathrm{CDCl}_{3}\right) 9.47$ $(1 \mathrm{H}, \mathrm{s},-\mathrm{CHO}), 7.83-7.77\left(2 \mathrm{H}, \mathrm{m}, \mathrm{SO}_{2}-\mathrm{C}-\mathrm{CH}\right), 7.33-7.28(2 \mathrm{H}, \mathrm{m}, \mathrm{Ar}-\mathbf{H}), 4.11(1 \mathrm{H}, \mathrm{d}$, J4.3, N-CH-C-CHO), $3.81\left(1 \mathrm{H}, \mathrm{d}, J 3.7, \mathrm{~N}-\mathrm{CH}-\mathrm{CH}-\mathrm{CH}_{3}\right), 2.43\left(3 \mathrm{H}, \mathrm{s}, \mathrm{Ar}-\mathrm{CH}_{3}\right), 2.09-$ $1.78\left(3 \mathrm{H}, \mathrm{m}, \mathrm{CH}_{2}-\mathrm{CHH}\right), 1.62-1.48\left(1 \mathrm{H}, \mathrm{m}, \mathrm{CH}_{2}-\mathrm{CHH}\right), 1.55\left(1 \mathrm{H}, \mathrm{q}, J 7.3, \mathrm{CH}-\mathrm{CH}_{3}\right)$, $1.03\left(3 \mathrm{H}, \mathrm{s}, \mathrm{CH}_{3}-\mathrm{C}-\mathrm{CHO}\right), 0.88\left(3 \mathrm{H}, \mathrm{d}, J 7.3, \mathrm{CH}-\mathrm{CH}_{3}\right) ; \delta_{\mathrm{C}}\left(125 \mathrm{MHz}, \mathrm{CDCl}_{3}\right) 204.5$ $(\mathrm{CH}), 143.8(\mathrm{C}), 137.3(\mathrm{C}), 129.7(\mathrm{CH}), 127.5(\mathrm{CH}), 66.0(\mathrm{CH}), 64.8(\mathrm{CH}), 56.2(\mathrm{C})$, $51.5(\mathrm{CH}), 30.0\left(\mathrm{CH}_{2}\right), 23.7\left(\mathrm{CH}_{2}\right), 21.6\left(\mathrm{CH}_{3}\right), 17.0\left(\mathrm{CH}_{3}\right), 16.0\left(\mathrm{CH}_{3}\right) ; \mathrm{m} / z(\mathrm{CI}) 308$ $\left(\mathrm{MH}^{+}, 100 \%\right)$; Found: $308.1330 . \mathrm{C}_{16} \mathrm{H}_{22} \mathrm{NO}_{3} \mathrm{~S}$ requires: 308.1320 . 
$( \pm)-\left(1 R^{*}, 3 S^{*}, 4 R^{*}, 5 S^{*}\right)-3,4-D i m e t h y l-8$-(toluene-4-sulfonyl)-8-aza-bicyclo[3.2.1]octan-2-one 8c

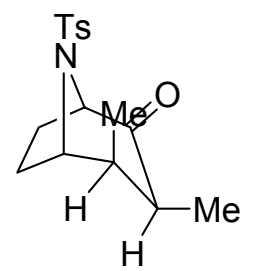

To a $0{ }^{\circ} \mathrm{C}$ solution of rearrangement precursor $\mathbf{6 c}(49 \mathrm{mg}, 0.14 \mathrm{mmol})$ in dichloromethane $(1 \mathrm{ml})$ was added $\mathrm{SnCl}_{4}$ solution $(0.28 \mathrm{ml}, 1.0 \mathrm{M}$ in heptanes, $0.28 \mathrm{mmol})$. The mixture was allowed to warm to ambient temperature and stirred for $16 \mathrm{~h}$. The reaction was quenched by addition of saturated aqueous $\mathrm{NaHCO}_{3}(10 \mathrm{ml})$. The mixture was extracted into chloroform $(2 \times 15 \mathrm{ml})$ and combined organics dried over $\mathrm{Na}_{2} \mathrm{SO}_{4}$. Flash Column Chromatography (eluant $3: 2$ petroleum : diethyl ether) isolated the product tropane 8c (21 mg, $49 \%$ ) as a colourless oil; $v_{\max } / \mathrm{cm}^{-1}$ (film) 2965, 2933, 2875, 1726; $\delta_{\mathrm{H}}(250$ $\left.\mathrm{MHz}_{\mathrm{CDCl}}\right)_{3}$ 7.78-7.72 (2H, m, SO $\left.2-\mathrm{C}-\mathrm{CH}\right), 7.30(2 \mathrm{H}, \mathrm{d}, J 7.9, \mathrm{Ar}-\mathbf{H}), 4.40-4.34(1 \mathrm{H}, \mathrm{m}$, Me-CH-CH-N), $4.03(1 \mathrm{H}, \mathrm{d}, J 7.6, \mathrm{~N}-\mathrm{CH}-\mathrm{C}=\mathrm{O}), 2.43\left(3 \mathrm{H}, \mathrm{s}, \operatorname{Ar}-\mathrm{CH}_{3}\right), 2.20(1 \mathrm{H}, \mathrm{dq}$, J6.8, 6.8, Me-CH-C=O), 1.87-1.57 (3H, m, CH $2-\mathrm{CHH}), 1.37-1.26\left(1 \mathrm{H}, \mathrm{m}, \mathrm{CH}_{2}-\mathrm{CHH}\right)$, $1.24\left(3 \mathrm{H}, \mathrm{d}, J 6.1, \mathrm{CH}_{3}-\mathrm{CH}-\mathrm{CH}-\mathrm{N}\right), 1.22-1.10(1 \mathrm{H}, \mathrm{m}, \mathrm{Me}-\mathrm{CH}-\mathrm{CH}-\mathrm{N}), 1.03$ (3H, d, J6.8, $\left.\mathrm{CH}_{3}-\mathrm{CH}-\mathrm{C}=\mathrm{O}\right) ; \delta_{\mathrm{C}}\left(125 \mathrm{MHz}, \mathrm{CDCl}_{3}\right) 211.8(\mathrm{C}), 144.0(\mathrm{C}), 136.9(\mathrm{C}), 129.9(\mathrm{CH})$, $127.4(\mathrm{CH}), 65.9(\mathrm{CH}), 62.7(\mathrm{CH}), 45.9(\mathrm{CH}), 43.3(\mathrm{CH}), 30.4\left(\mathrm{CH}_{2}\right), 29.0\left(\mathrm{CH}_{2}\right), 21.6$ $\left(\mathrm{CH}_{3}\right), 21.1\left(\mathrm{CH}_{3}\right), 13.0\left(\mathrm{CH}_{3}\right) ; \mathrm{m} / z(\mathrm{CI}) 325\left[\mathrm{M}\left(\mathrm{NH}_{4}\right)^{+}, 100 \%\right], 308\left(\mathrm{MH}^{+}, 35 \%\right)$; Found: $\left(\mathrm{MH}^{+}\right)$308.1323. $\mathrm{C}_{16} \mathrm{H}_{22} \mathrm{NO}_{3} \mathrm{~S}$ requires: 308.1320 . 
$( \pm)-\left(1 R^{*}, \quad 2 S^{*}, \quad 3 S^{*}, \quad 4 S^{*}\right)-($ cis-2,3-Dimethyl)-7-(toluene-4-sulfonyl)-7-aza-bicyclo[2.2.1] carbaldehyde $7 \mathbf{d}$

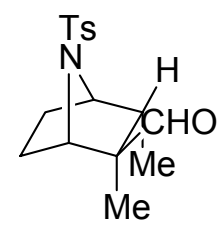

A solution of $(E)$-2-bromo-but-2-ene $(0.16 \mathrm{ml}, 1.55 \mathrm{mmol})$ in diethyl ether $(10 \mathrm{ml})$ was cooled to $-78{ }^{\circ} \mathrm{C}$. ${ }^{\mathrm{t}} \mathrm{BuLi}(1.82 \mathrm{ml}, 1.7 \mathrm{M}$ in pentane, $3.10 \mathrm{mmol})$ was added and the mixture stirred at $-78{ }^{\circ} \mathrm{C}$ for $1 \mathrm{~h}$. Solid $\mathrm{MgBr}_{2} . \mathrm{OEt}_{2}(364 \mathrm{mg}, 1.41 \mathrm{mmol}$ ) was introduced and the temperature raised to $0{ }^{\circ} \mathrm{C}$ for $30 \mathrm{~min}$. Separately, the aldehyde $\mathbf{5 a}(351 \mathrm{mg}, 1.24$ mmol) was dissolved in diethyl ether $(7 \mathrm{ml})$ and the whole cooled to $0{ }^{\circ} \mathrm{C}$. The entire organometallic solution (at $0^{\circ} \mathrm{C}$ ) was transferred into this via canula with stirring. Stirred a further $5 \mathrm{~min}$ at ambient temperature before quenching with saturated aqueous $\mathrm{NH}_{4} \mathrm{Cl}$ $(20 \mathrm{ml})$. The mixture was extracted into diethyl ether $(2 \times 20 \mathrm{ml})$ and combined organics dried over $\mathrm{Na}_{2} \mathrm{SO}_{4}$. Flash Column Chromatography (eluant $3: 2$ petroleum : diethyl ether) isolated the desired rearrangement precursor $\mathbf{6 d}$ as a mixture of diastereoisomers $(252 \mathrm{mg}, 60 \%)$ as a colourless oil; $v_{\max } / \mathrm{cm}^{-1}$ (film) 3497, 2980, 2937, 2865, 2835, 1598; $\mathrm{m} / \mathrm{z}(\mathrm{CI}) 357\left[\mathrm{M}\left(\mathrm{NH}_{4}\right)^{+}, 10 \%\right], 308\left[\left(\mathrm{MH}^{+}\right)-\mathrm{MeOH}, 100 \%\right]$; Found: 357.1846 $\left[\mathrm{M}\left(\mathrm{NH}_{4}\right)^{+}\right] . \mathrm{C}_{17} \mathrm{H}_{29} \mathrm{~N}_{2} \mathrm{O}_{4} \mathrm{~S}$ requires: 357.1848 .

To a $0{ }^{\circ} \mathrm{C}$ solution of rearrangement precursor $\mathbf{6 d}(72 \mathrm{mg}, 0.21 \mathrm{mmol})$ in dichloromethane $(1.5 \mathrm{ml})$ was added $\mathrm{SnCl}_{4}$ solution $(0.21 \mathrm{ml}, 1.0 \mathrm{M}$ in heptanes, 0.21 $\mathrm{mmol}$ ). The whole was stirred at $0{ }^{\circ} \mathrm{C}$ for $8 \mathrm{~min}$ before being quenched by addition of saturated aqueous $\mathrm{NaHCO}_{3}(7 \mathrm{ml})$. The mixture was extracted into dichloromethane $(2 \mathrm{x}$ $15 \mathrm{ml}$ ) and combined organics dried over $\mathrm{Na}_{2} \mathrm{SO}_{4}$. Flash Column Chromatography (eluant $3: 2$ petroleum : diethyl ether) afforded the aldehyde $7 \mathbf{d}(40 \mathrm{mg}, 61 \%)$ as white crystals, m.p. $126-128{ }^{\circ} \mathrm{C} ; v_{\max } / \mathrm{cm}^{-1}$ (film) $2971,2929,2882,1721,1597 ; \delta_{\mathrm{H}}(250 \mathrm{MHz}$, $\left.\mathrm{CDCl}_{3}\right) 9.38(1 \mathrm{H}, \mathrm{s},-\mathrm{CHO}), 7.78-7.72\left(2 \mathrm{H}, \mathrm{m}, \mathrm{SO}_{2}-\mathrm{C}-\mathrm{CH}\right), 7.28(2 \mathrm{H}, \mathrm{d}, J 7.9, \mathrm{Ar}-\mathbf{H})$, $4.14(1 \mathrm{H}, \mathrm{d}, J 4.6, \mathrm{~N}-\mathrm{CH}-\mathrm{C}-\mathrm{CHO}), 4.09-4.04\left(1 \mathrm{H}, \mathrm{m}, \mathrm{N}-\mathrm{CH}-\mathrm{CH}-\mathrm{CH}_{3}\right), 2.48(1 \mathrm{H}, \mathrm{qd}$, J7.5, 4.6, CH-CH $\left.{ }_{3}\right), 2.42\left(3 \mathrm{H}, \mathrm{s}, \mathrm{Ar}-\mathrm{CH}_{3}\right), 1.97-1.71\left(4 \mathrm{H}, \mathrm{m}, \mathrm{CH}_{2}-\mathrm{CH}_{2}\right), 0.90(3 \mathrm{H}, \mathrm{s}$, $\left.\mathrm{CH}_{3}-\mathrm{C}-\mathrm{CHO}\right), 0.86\left(3 \mathrm{H}, \mathrm{d}, J 7.5, \mathrm{CH}-\mathrm{CH}_{3}\right) ; \delta_{\mathrm{C}}\left(125 \mathrm{MHz}, \mathrm{CDCl}_{3}\right) 202.7(\mathrm{CH}), 143.7(\mathrm{C})$, $137.4(\mathrm{C}), 129.6(\mathrm{CH}), 127.5(\mathrm{CH}), 65.0(\mathrm{CH}), 64.3(\mathrm{CH}), 54.5(\mathrm{C}), 38.1(\mathrm{CH}), 23.7$ $\left(\mathrm{CH}_{2}\right), 22.8\left(\mathrm{CH}_{2}\right), 21.5\left(\mathrm{CH}_{3}\right), 11.6\left(\mathrm{CH}_{3}\right), 10.7\left(\mathrm{CH}_{3}\right) ; \mathrm{m} / z(\mathrm{CI}) 308\left(\mathrm{MH}^{+}, 100 \%\right)$; Found: $\left(\mathrm{MH}^{+}\right)$308.1332. $\mathrm{C}_{16} \mathrm{H}_{22} \mathrm{NO}_{3} \mathrm{~S}$ requires: 308.1320 . 
$( \pm)-\left(1 R^{*}, 3 S^{*}, 4 S^{*}, 5 S^{*}\right)-3,4$-Dimethyl-8-(toluene-4-sulfonyl)-8-aza-bicyclo[3.2.1]octan-2-one 8d

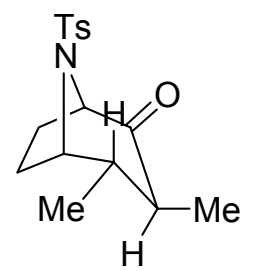

To a $0{ }^{\circ} \mathrm{C}$ solution of rearrangement precursor $\mathbf{6 d}(63 \mathrm{mg}, 0.19 \mathrm{mmol})$ in dichloromethane $(1.2 \mathrm{ml})$ was added $\mathrm{SnCl}_{4}$ solution $(0.37 \mathrm{ml}, 1.0 \mathrm{M}$ in heptanes, 0.37 mmol). The mixture was allowed to warm to ambient temperature and stirred for $16 \mathrm{~h}$. The reaction was quenched by addition of saturated aqueous $\mathrm{NaHCO}_{3}(10 \mathrm{ml})$. The mixture was extracted into chloroform $(2 \times 15 \mathrm{ml})$ and combined organics dried over $\mathrm{Na}_{2} \mathrm{SO}_{4}$. Flash Column Chromatography (eluant $3: 2$ petroleum : diethyl ether) isolated the product tropane $8 \mathbf{d}(24 \mathrm{mg}, 42 \%)$ as a white solid, m.p. $107-110{ }^{\circ} \mathrm{C} ; v_{\max } / \mathrm{cm}^{-1}$ (film) 2971, 2933, 2882, 1724, 1597; $\delta_{\mathrm{H}}\left(250 \mathrm{MHz}, \mathrm{CDCl}_{3}\right)$ 7.76-7.70 (2H, m, $\mathrm{SO}_{2}-\mathrm{C}-$ CH), 7.29 (2H, d, J8.2, Ar-H), $4.26(1 \mathrm{H}, \mathrm{d}, J 7.0, \mathrm{~N}-\mathrm{CH}-\mathrm{C}=\mathrm{O}), 4.17(1 \mathrm{H}, \mathrm{dd}, J 7.3,3.7$, Me-CH-CH-N), 2.43 (3H, s, Ar-CH $)_{3}, 2.05-1.56$ (6H, m, CH$\left.-\mathrm{CH}_{2}, \mathrm{Me}-\mathrm{CH}-\mathrm{CH}-\mathrm{Me}\right)$, $1.00\left(3 \mathrm{H}, \mathrm{d}, J 6.4, \mathrm{CH}_{3}-\mathrm{CH}-\mathrm{CH}-\mathrm{N}\right), 1.00\left(3 \mathrm{H}, \mathrm{d}, J 6.4, \mathrm{CH}_{3}-\mathrm{CH}-\mathrm{C}=\mathrm{O}\right) ; \delta_{\mathrm{C}}(100 \mathrm{MHz}$, $\left.\mathrm{CDCl}_{3}\right) 204.2(\mathrm{CH}), 144.0(\mathrm{C}), 136.7(\mathrm{C}), 129.8(\mathrm{CH}), 127.5(\mathrm{CH}), 65.3(\mathrm{CH}), 62.1(\mathrm{CH})$, $44.0(\mathrm{CH}), 43.9(\mathrm{CH}), 27.7\left(\mathrm{CH}_{2}\right), 22.4\left(\mathrm{CH}_{2}\right), 21.6\left(\mathrm{CH}_{3}\right), 16.2\left(\mathrm{CH}_{3}\right), 11.5\left(\mathrm{CH}_{3}\right) ; \mathrm{m} / \mathrm{z}$ (CI) $325\left[\mathrm{M}\left(\mathrm{NH}_{4}\right)^{+}, 100 \%\right], 308\left(\mathrm{MH}^{+}, 35 \%\right)$; Found: $\left(\mathrm{MH}^{+}\right)$308.1320. $\mathrm{C}_{16} \mathrm{H}_{22} \mathrm{NO}_{3} \mathrm{~S}$ requires: 308.1320 . 
$( \pm)-\left(1 R^{*}, 2 S^{*}, 4 S^{*}\right)-7-(T o l u e n e-4-s u l f o n y l)-7-a z a-b i c y c l o[2.2 .1]$ heptane-2-carbaldehyde 7e<smiles>O=C1CC2CCC1N2[As]</smiles>

To a solution of the aldehyde $\mathbf{5 a}(380 \mathrm{mg}, 1.34 \mathrm{mmol})$ in diethyl ether $(10 \mathrm{ml})$ at $-30{ }^{\circ} \mathrm{C}$ was added vinyl magnesium bromide solution $(1.61 \mathrm{ml}, 1.0 \mathrm{M}, 1.61 \mathrm{mmol})$. The reaction was allowed to warm to ambient temperature for $10 \mathrm{~min}$ before being quenched by addition of saturated aqueous $\mathrm{NH}_{4} \mathrm{Cl}$ solution $(20 \mathrm{ml})$. The mixture was extracted into diethyl ether $(2 \times 30 \mathrm{ml})$, the combined organics washed with brine and then dried over $\mathrm{Na}_{2} \mathrm{SO}_{4}$. Flash Column Chromatography (eluant $3: 2$ petroleum : diethyl ether, gradient rising to $1: 1$ ) isolated the rearrangement precursor $\mathbf{6 e}$ as a mixture of diastereoisomers (236 mg, $56 \%$ ) as a colourless oil; $v_{\max } / \mathrm{cm}^{-1}$ (film) 3541, 3069, 2986, 2955, 2835, 1598, $1557 ; \mathrm{m} / \mathrm{z}(\mathrm{CI}) 329\left[\mathrm{M}\left(\mathrm{NH}_{4}\right)^{+}, 20 \%\right], 280\left[\left(\mathrm{MH}^{+}\right)-\mathrm{MeOH}, 100 \%\right.$ ]; Found: 329.1544 $\left[\mathrm{M}\left(\mathrm{NH}_{4}\right)^{+}\right] . \mathrm{C}_{15} \mathrm{H}_{25} \mathrm{~N}_{2} \mathrm{O}_{4} \mathrm{~S}$ requires: 329.1535 .

To a $0{ }^{\circ} \mathrm{C}$ solution of rearrangement precursor $6 \mathrm{e}(34 \mathrm{mg}, 0.11 \mathrm{mmol})$ in dichloromethane $(1 \mathrm{ml})$ was added $\mathrm{SnCl}_{4}$ solution $(0.11 \mathrm{ml}, 1.0 \mathrm{M}$ in heptanes, $0.11 \mathrm{mmol})$. The mixture was allowed to warm to ambient temperature and stirred for $14.5 \mathrm{~h}$, before being quenched by addition of saturated aqueous $\mathrm{NaHCO}_{3}(5 \mathrm{ml})$. The mixture was extracted into dichloromethane $(2 \times 10 \mathrm{ml})$ and combined organics dried over $\mathrm{Na}_{2} \mathrm{SO}_{4}$. Flash Column Chromatography (eluant $2: 3$ petroleum : diethyl ether, gradient rising to $1: 2$ ) afforded the aldehyde 7e (10 mg, $32 \%$ ) as a white gum; $v_{\max } / \mathrm{cm}^{-1}$ (film) 2956, 2925, $2881,1722,1598 ; \delta_{\mathrm{H}}\left(250 \mathrm{MHz}, \mathrm{CDCl}_{3}\right) 9.49(1 \mathrm{H}, \mathrm{d}, J 1.8,-\mathrm{CHO}), 7.78-7.72(2 \mathrm{H}, \mathrm{m}$, $\mathrm{SO}_{2}$-C-CH), 7.31-7.26 (2H, m, Ar-H), 4.44 (1H, d, J4.0, N-CH-CH-CHO), 4.29 [1H, t, J4.9, N-CH- $\left.\left(\mathrm{CH}_{2}\right)_{2}\right], 2.48-2.41(1 \mathrm{H}, \mathrm{m}, \mathrm{CH}-\mathrm{CHO}), 2.42\left(3 \mathrm{H}, \mathrm{s}, \mathrm{Ar}-\mathrm{CH}_{3}\right), 2.27-2.16(1 \mathrm{H}$, $\mathrm{m}$, exo -CHH-C-CHO), 2.03-1.95 (2H, m, -CHH-CHH- both exo), 1.70-1.50 (3H, m, CHH-CHH- and -CHH-C-CHO, all endo); $\delta_{\mathrm{C}}\left(100 \mathrm{MHz}, \mathrm{CDCl}_{3}\right) 200.7(\mathrm{CH}), 143.9(\mathrm{C})$, $137.1(\mathrm{C}), 129.6(\mathrm{CH}), 127.6(\mathrm{CH}), 60.0(\mathrm{CH}), 58.9(\mathrm{CH}), 54.5(\mathrm{CH}), 32.1\left(\mathrm{CH}_{2}\right), 29.9$ $\left(\mathrm{CH}_{2}\right), 29.1\left(\mathrm{CH}_{2}\right), 21.6\left(\mathrm{CH}_{3}\right) ; \mathrm{m} / z(\mathrm{CI}) 280\left(\mathrm{MH}^{+}, 100 \%\right)$; Found: 280.0999. $\mathrm{C}_{14} \mathrm{H}_{18} \mathrm{NO}_{3} \mathrm{~S}$ requires: 280.1007 . 
( \pm$)-\left(1 R^{*}, 2 R^{*}, 4 S^{*}\right)$-2-Phenylsulfanyl-7-(toluene-4-sulfonyl)-7-aza-bicyclo[2.2.1] heptane-2carbaldehyde $7 f$<smiles>O=CC1(c2ccccc2)C2CCC(C2)N1[SH+]</smiles>

A solution of TMEDA $(0.24 \mathrm{ml}, 1.57 \mathrm{mmol})$ in tetrahydrofuran $(6 \mathrm{ml})$ was cooled to -78 ${ }^{\circ} \mathrm{C}$ and ${ }^{\mathrm{n}} \mathrm{BuLi}(0.63 \mathrm{ml}, 2.5 \mathrm{M}, 1.57 \mathrm{mmol})$ added. A solution of phenylthioethylene (214 $\mathrm{mg}, 1.57 \mathrm{mmol})$ in tetrahydrofuran $(4.5 \mathrm{ml})$ was added over $10 \mathrm{~min}$. The mixture was stirred a further $30 \mathrm{~min}$ at $-78{ }^{\circ} \mathrm{C}$. Solid $\mathrm{MgBr}_{2} . \mathrm{OEt}_{2}(405 \mathrm{mg}, 1.57 \mathrm{mmol}$ ) was added and the reaction warmed to $0{ }^{\circ} \mathrm{C}$, causing dissolution of the solid. Separately, the aldehyde 5a $(365 \mathrm{mg}, 1.29 \mathrm{mmol})$ was dissolved in tetrahydrofuran $(6 \mathrm{ml})$ and the solution cooled to $30{ }^{\circ} \mathrm{C}$. The entire organometallic solution was transferred into this via canula and the resulting mixture warmed to ambient temperature over $15 \mathrm{~min}$. The reaction was quenched by addition of saturated aqueous $\mathrm{NH}_{4} \mathrm{Cl}(30 \mathrm{ml})$ and extracted into diethyl ether $(2 \times 30 \mathrm{ml})$. The combined organics were dried over $\mathrm{Na}_{2} \mathrm{SO}_{4}$ and Flash Column Chromatography (eluant $3: 2$ petroleum : diethyl ether) isolated sulfide 6f, a mixture of diastereoisomers (205 mg, $38 \%$ ), as a colourless oil; $m / z(\mathrm{CI}) 437\left[\mathrm{M}\left(\mathrm{NH}_{4}\right)^{+}, 85 \%\right)$; Found: 437.1576. $\mathrm{C}_{21} \mathrm{H}_{29} \mathrm{~N}_{2} \mathrm{O}_{4} \mathrm{~S}_{2}$ requires: 437.1569 .

To a $0{ }^{\circ} \mathrm{C}$ solution of sulfide $\mathbf{6 f}(38 \mathrm{mg}, 0.091 \mathrm{mmol})$ in dichloromethane $(1 \mathrm{ml})$ was added $\mathrm{SnCl}_{4}$ solution $(91 \mu \mathrm{l}, 1.0 \mathrm{M}$ in heptanes, $0.091 \mathrm{mmol})$. The whole was stirred at 0 ${ }^{\circ} \mathrm{C}$ for $1 \mathrm{~min}$ before being quenched by addition of saturated aqueous $\mathrm{NaHCO}_{3}(5 \mathrm{ml})$. The mixture was extracted into dichloromethane $(2 \times 10 \mathrm{ml})$, dried over $\mathrm{Na}_{2} \mathrm{SO}_{4}$, filtered and solvent removed in vacuo to afford a crude residue. Flash Column Chromatography (eluant 1: 1 petroleum : diethyl ether) isolated the aldehyde 7f (4 mg, $11 \%$ ) as a colourless oil; $v_{\max } / \mathrm{cm}^{-1}$ (film) 3062, 2960, 1721, 1597; $\delta_{\mathrm{H}}\left(250 \mathrm{MHz}, \mathrm{CDCl}_{3}\right) 9.10(1 \mathrm{H}$, s, -CHO), 7.67 (2H, d, J8.2, $\left.\mathrm{SO}_{2}-\mathrm{C}-\mathrm{CH}\right), 7.39-7.21(7 \mathrm{H}, \mathrm{m}, \mathrm{Ar}-\mathrm{H}), 4.41-4.36(1 \mathrm{H}, \mathrm{m}, \mathrm{N}-$ CH-C-CHO), 4.27-4.21 [1H, m, N-CH( $\left.\left(\mathrm{CH}_{2}\right)_{2}\right], 2.69-2.55\left(1 \mathrm{H}, \mathrm{m}\right.$, endo -CHH-CH $\left.2^{-}\right)$, 2.54-2.40 (1H, m, exo -CHH-C-CHO), $2.40\left(3 \mathrm{H}, \mathrm{s}, \mathrm{Ar}-\mathrm{CH}_{3}\right), 2.19-2.00(2 \mathrm{H}, \mathrm{m},-\mathrm{CH}-$ CHH- both exo $)$, 1.74-1.61 (1H, m, endo -CHH-CH $\left.2^{-}\right), 1.24(1 \mathrm{H}, \mathrm{d}, \mathrm{J12} .8$, endo -CHH$\mathrm{C}-\mathrm{CHO}) ; \delta_{\mathrm{C}}\left(125 \mathrm{MHz}, \mathrm{CDCl}_{3}\right) 192.0(\mathrm{CH}), 143.8(\mathrm{C}), 137.2(\mathrm{C}), 136.6(\mathrm{C}), 134.9(\mathrm{CH})$, $129.5(\mathrm{CH}), 129.3(\mathrm{CH}), 129.3(\mathrm{CH}), 127.5(\mathrm{CH}), 64.8(\mathrm{C}), 62.3(\mathrm{CH}), 59.6(\mathrm{CH}), 38.7$ $\left(\mathrm{CH}_{2}\right), 30.6\left(\mathrm{CH}_{2}\right), 24.2\left(\mathrm{CH}_{2}\right), 21.5\left(\mathrm{CH}_{3}\right) ; \mathrm{m} / \mathrm{z}(\mathrm{CI}) 388\left(\mathrm{MH}^{+}, 100 \%\right)$; Found: 388.1031. $\mathrm{C}_{20} \mathrm{H}_{22} \mathrm{NO}_{3} \mathrm{~S}_{2}$ requires: 388.1041. 
$( \pm)-\left(1 R^{*}, 3 S^{*}, 4 R^{*}\right)-3-(1-B e n z e n e s u l f o n y l-v i n y l)-7-(t o l u e n e-4-s u l f o n y l)-2-o x a-7-a z a-$

bicyclo[2.2.1]heptane 10

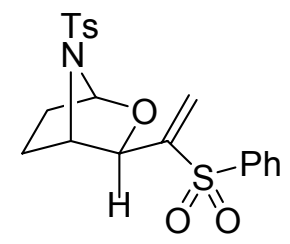

To a solution of sulfide $\mathbf{6 f}(71 \mathrm{mg}, 0.17 \mathrm{mmol})$ in dichloromethane $(5 \mathrm{ml})$ was added $\mathrm{NaHCO}_{3}(36 \mathrm{mg}, 0.43 \mathrm{mmol})$ and the mixture cooled to $-78^{\circ} \mathrm{C} . \mathrm{m}$-chloroperbenzoic acid solution $(0.38 \mathrm{M}$ in dichloromethane as determined by iodometric titration, $0.89 \mathrm{ml}, 0.34$ mmol) was added and the whole stirred at $-78{ }^{\circ} \mathrm{C}$ for $2 \mathrm{~h}$. The reaction was warmed to ambient temperature and stirred for $17 \mathrm{~h}$ before being quenched by addition of saturated aqueous $\mathrm{NaHCO}_{3}(20 \mathrm{ml})$. Extraction into chloroform $(2 \times 20 \mathrm{ml})$, drying over $\mathrm{Na}_{2} \mathrm{SO}_{4}$ and removal of solvent in vacuo gave a crude residue which was purified by Flash Column Chromatography (eluant 2:3 petroleum : diethyl ether) to afford the desired sulfone $6 \mathbf{h}(50 \mathrm{mg}, 65 \%)$ as a mixture of diastereoisomers; $m / z(\mathrm{CI}) 469\left[\mathrm{M}\left(\mathrm{NH}_{4}\right)^{+}, 5 \%\right.$ ], $420\left[\left(\mathrm{MH}^{+}\right)-\mathrm{MeOH}, 50 \%\right]$; Found $\left[\mathrm{M}\left(\mathrm{NH}_{4}\right)^{+}\right]:$469.1447. $\mathrm{C}_{21} \mathrm{H}_{29} \mathrm{~N}_{2} \mathrm{O}_{6} \mathrm{~S}_{2}$ requires: 469.1467.

To a $0{ }^{\circ} \mathrm{C}$ solution of sulfone $6 \mathbf{h}(23 \mathrm{mg}, 0.051 \mathrm{mmol})$ in dichloromethane $(1 \mathrm{ml})$ was added $\mathrm{SnCl}_{4}$ solution $(51 \mu \mathrm{l}, 1.0 \mathrm{M}$ in heptanes, $0.051 \mathrm{mmol})$. The whole was stirred at 0 ${ }^{\circ} \mathrm{C}$ for $8 \mathrm{~min}$ before being quenched by addition of saturated aqueous $\mathrm{NaHCO}_{3}(5 \mathrm{ml})$. The mixture was extracted into dichloromethane $(2 \times 10 \mathrm{ml})$, dried over $\mathrm{Na}_{2} \mathrm{SO}_{4}$, filtered and solvent removed in vacuo to afford a crude residue. Flash Column Chromatography (eluant 2 : 3 petroleum : diethyl ether) isolated the bicycle $10(14 \mathrm{mg}, 66 \%)$ as an amorphous powder; $v_{\max } / \mathrm{cm}^{-1}$ (film) 3060, 2953, 1761, 1598; $\delta_{\mathrm{H}}\left(250 \mathrm{MHz}, \mathrm{CDCl}_{3}\right)$ 8.25-8.17 (2H, m, Ar-H), 7.71-7.64 (3H, m, Ar-H), 7.57 (2H, d, J8.2, $\left.\mathrm{SO}_{2}-\mathrm{C}-\mathrm{CH}\right), 7.23$ $(2 \mathrm{H}, \mathrm{d}, J 8.2, \mathrm{Ar}-\mathbf{H}), 6.53(1 \mathrm{H}, \mathrm{s}, \mathrm{C}=\mathrm{CHH}), 6.05(1 \mathrm{H}, \mathrm{s}, \mathrm{C}=\mathrm{CHH}), 5.18(1 \mathrm{H}, \mathrm{s}, \mathrm{O}-\mathrm{CH}-$ $\mathrm{C}=\mathrm{C}), 4.57(1 \mathrm{H}, \mathrm{d}, J 4.6$, N-CH-O), 4.40-4.31 (1H, m, CH-CH-C=C), $2.38(3 \mathrm{H}, \mathrm{s}, \mathrm{Ar}-$ $\left.\mathrm{CH}_{3}\right)$, 2.23-2.09 [1H, m, endo $\left.\mathrm{CHH}-\mathrm{CH}(\mathrm{N})-\mathrm{CH}\right], 1.51-1.41[1 \mathrm{H}, \mathrm{m}$, endo $\mathrm{CHH}-\mathrm{CH}(\mathrm{N})-$ O], 1.27-1.16 [1H, m, exo CHH-CH(N)-CH], 0.83-0.67 [1H, m, exo CHH-CH(N)-O]; $\delta_{\mathrm{C}}$ $\left(125 \mathrm{MHz}, \mathrm{CDCl}_{3}\right) 150.6(\mathrm{C}), 143.2(\mathrm{C}), 138.3(\mathrm{C}), 135.8(\mathrm{C}), 133.5(\mathrm{CH}), 129.7(\mathrm{CH})$, 129.6 (CH), $129.1(\mathrm{CH}), 127.5(\mathrm{CH}), 126.2\left(\mathrm{CH}_{2}\right), 90.4(\mathrm{CH}), 73.1(\mathrm{CH}), 62.8(\mathrm{CH}), 32.5$ $\left(\mathrm{CH}_{2}\right), 21.5\left(\mathrm{CH}_{3}\right), 20.9\left(\mathrm{CH}_{2}\right) ; \mathrm{m} / z(\mathrm{CI}) 437\left[\mathrm{M}\left(\mathrm{NH}_{4}\right)^{+}, 50 \%\right]$; Found $\left(\mathrm{MH}^{+}\right): 420.0927$. $\mathrm{C}_{20} \mathrm{H}_{22} \mathrm{NO}_{5} \mathrm{~S}_{2}$ requires: 420.0939 . 


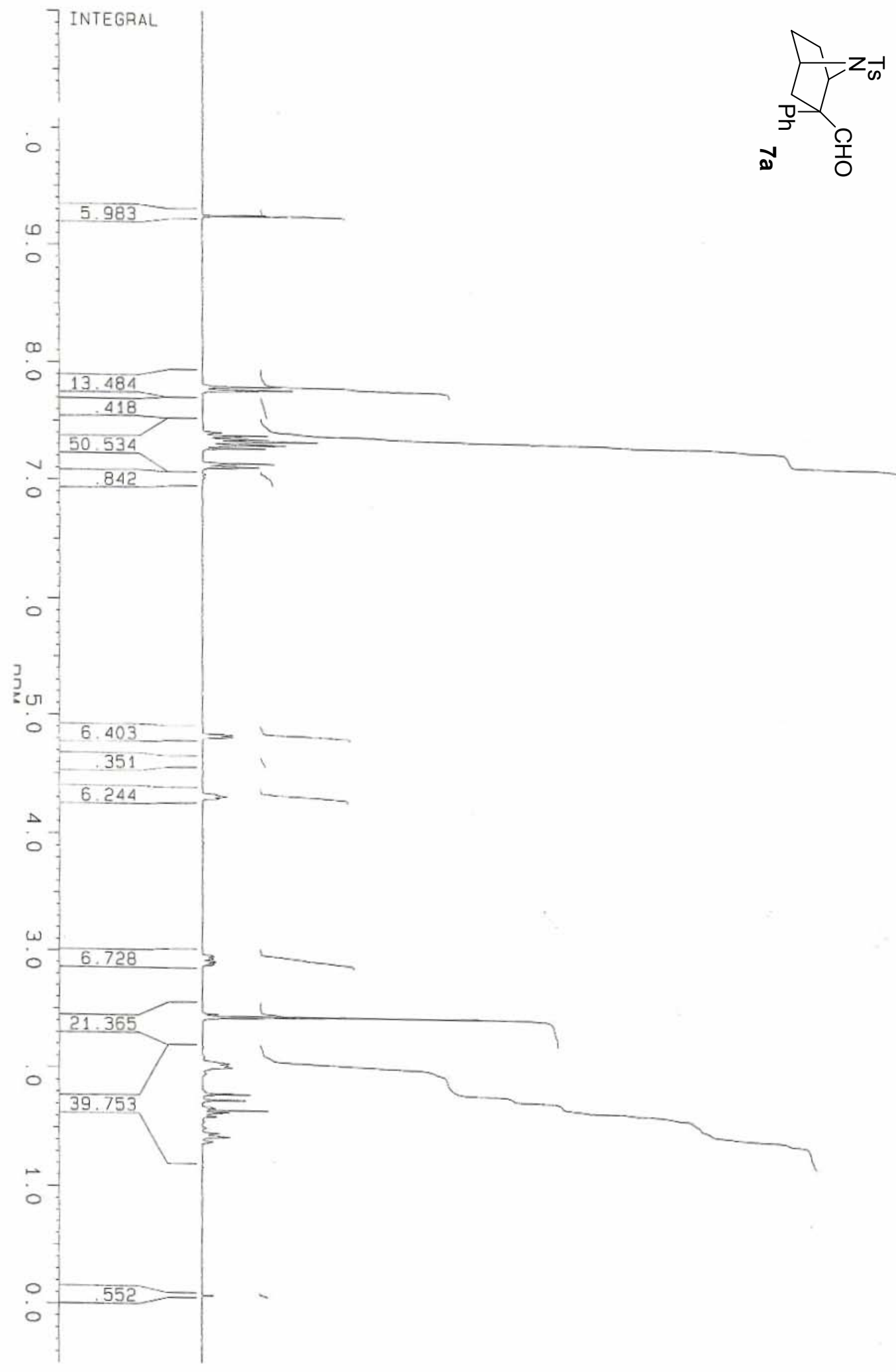




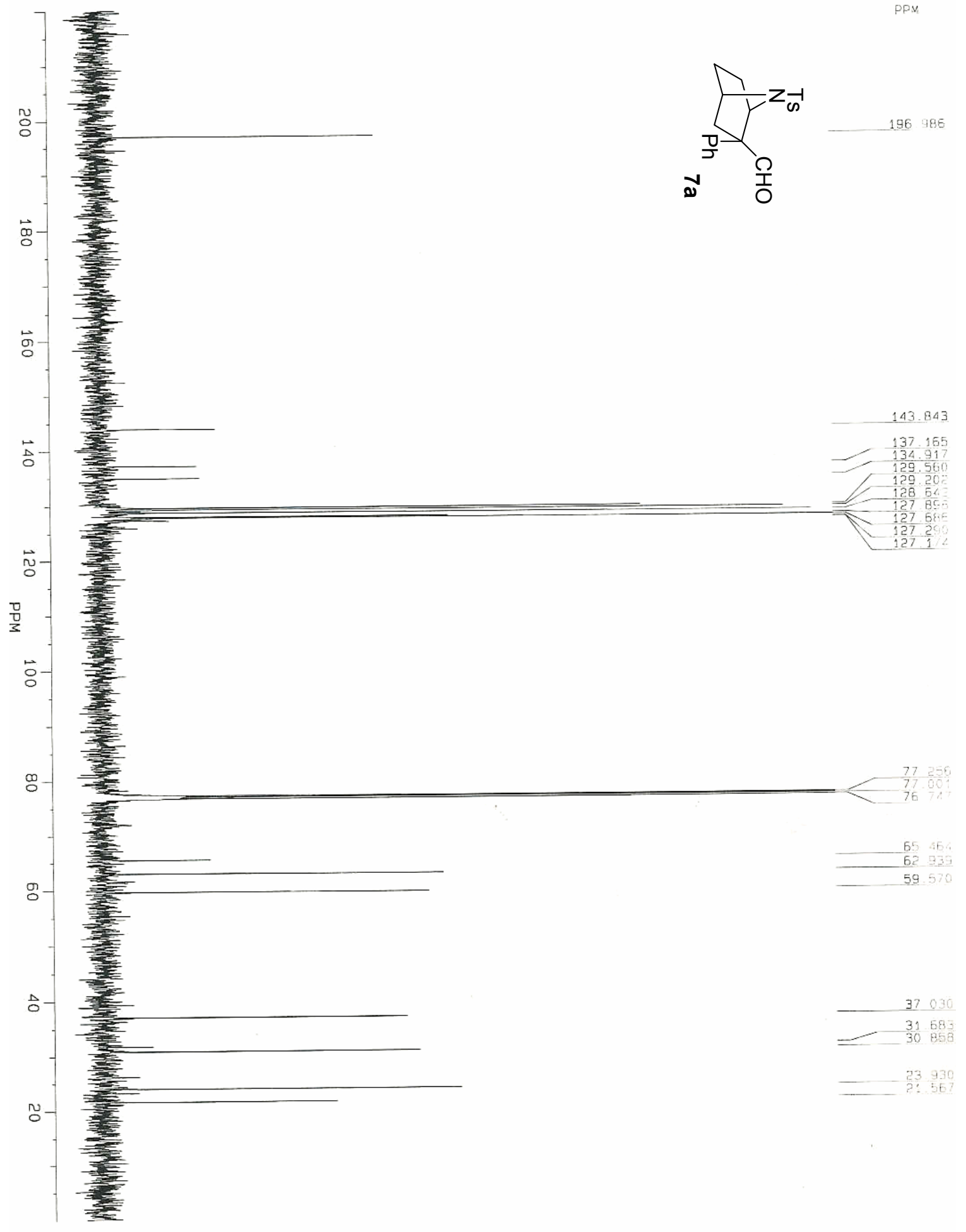




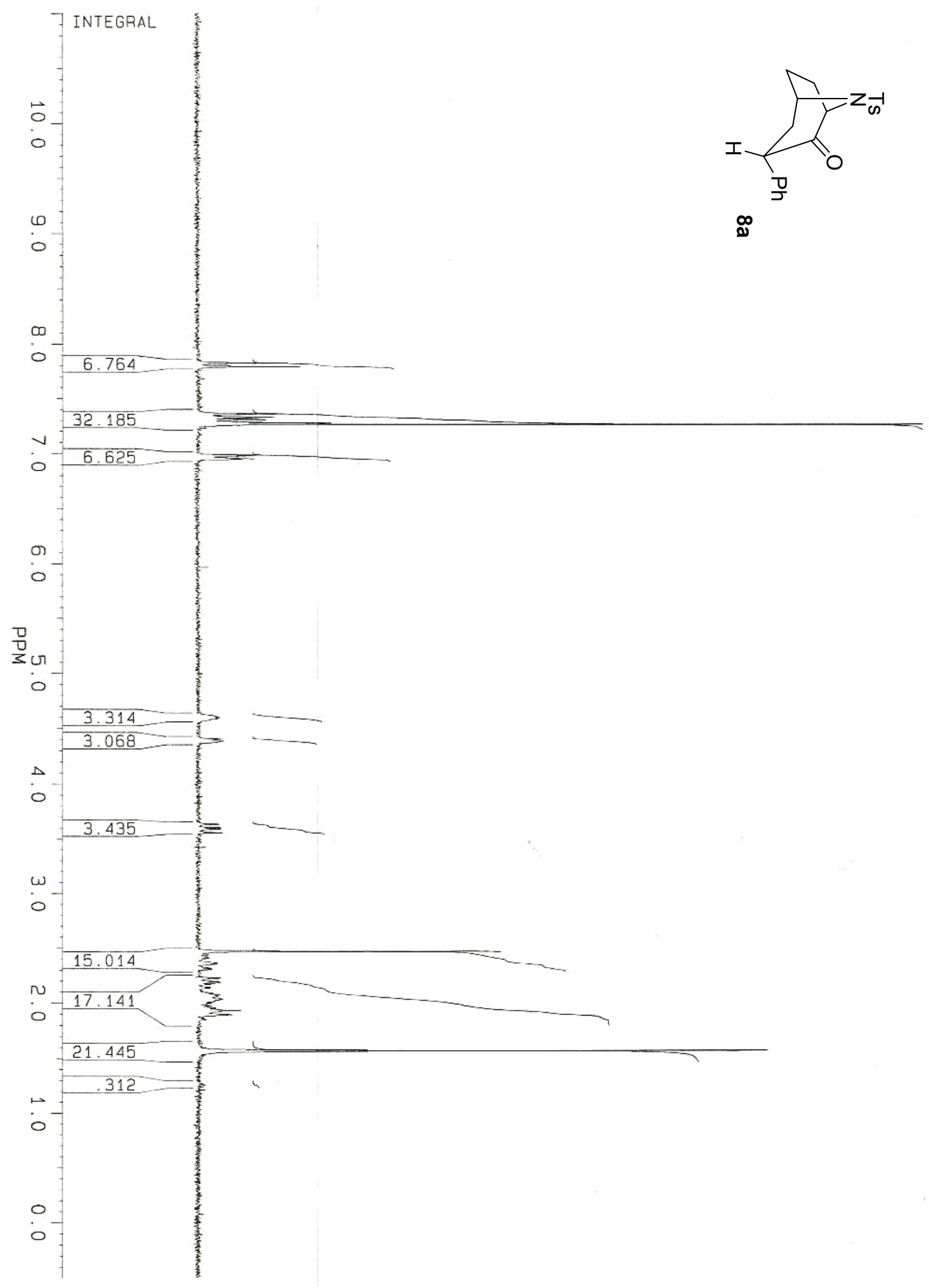




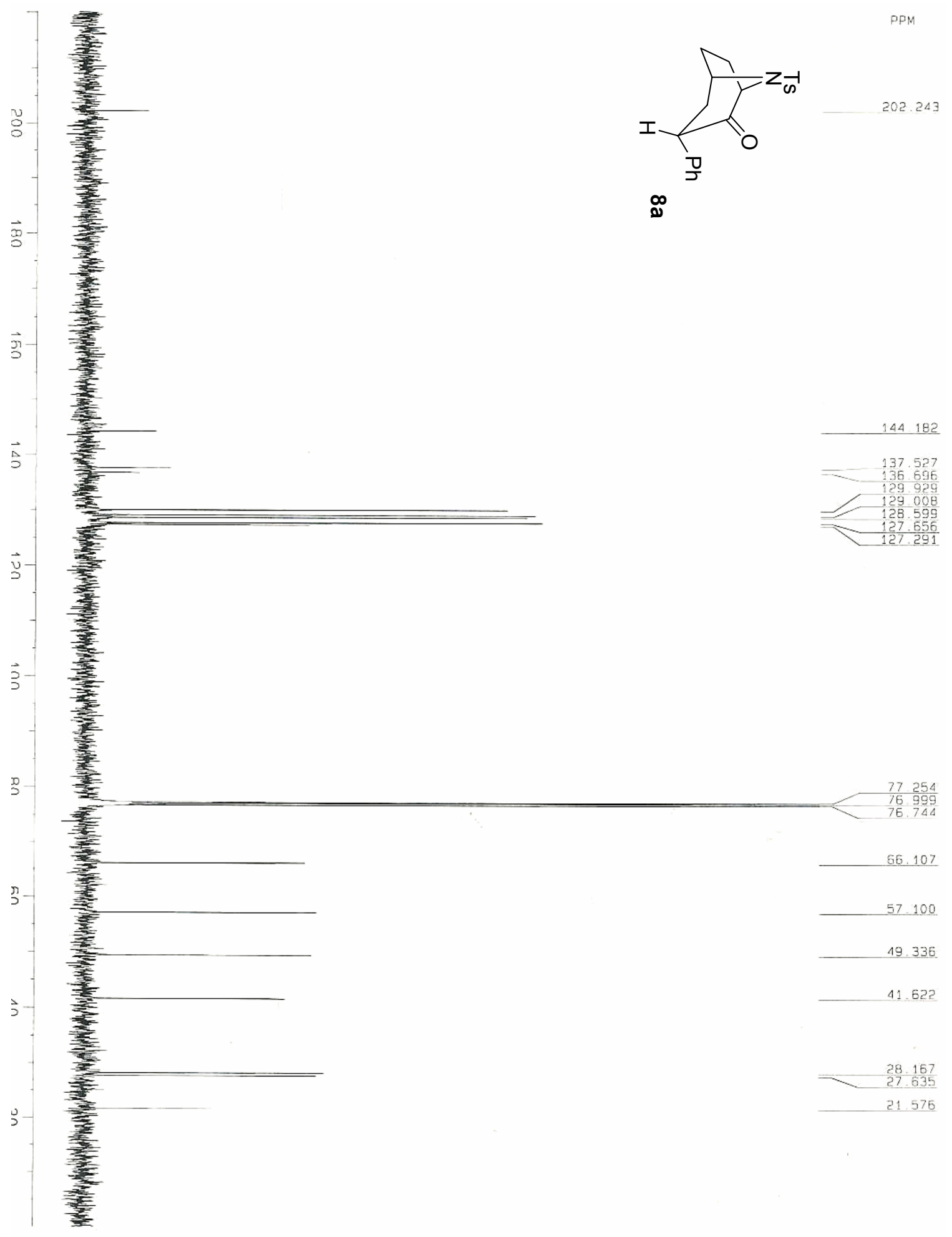




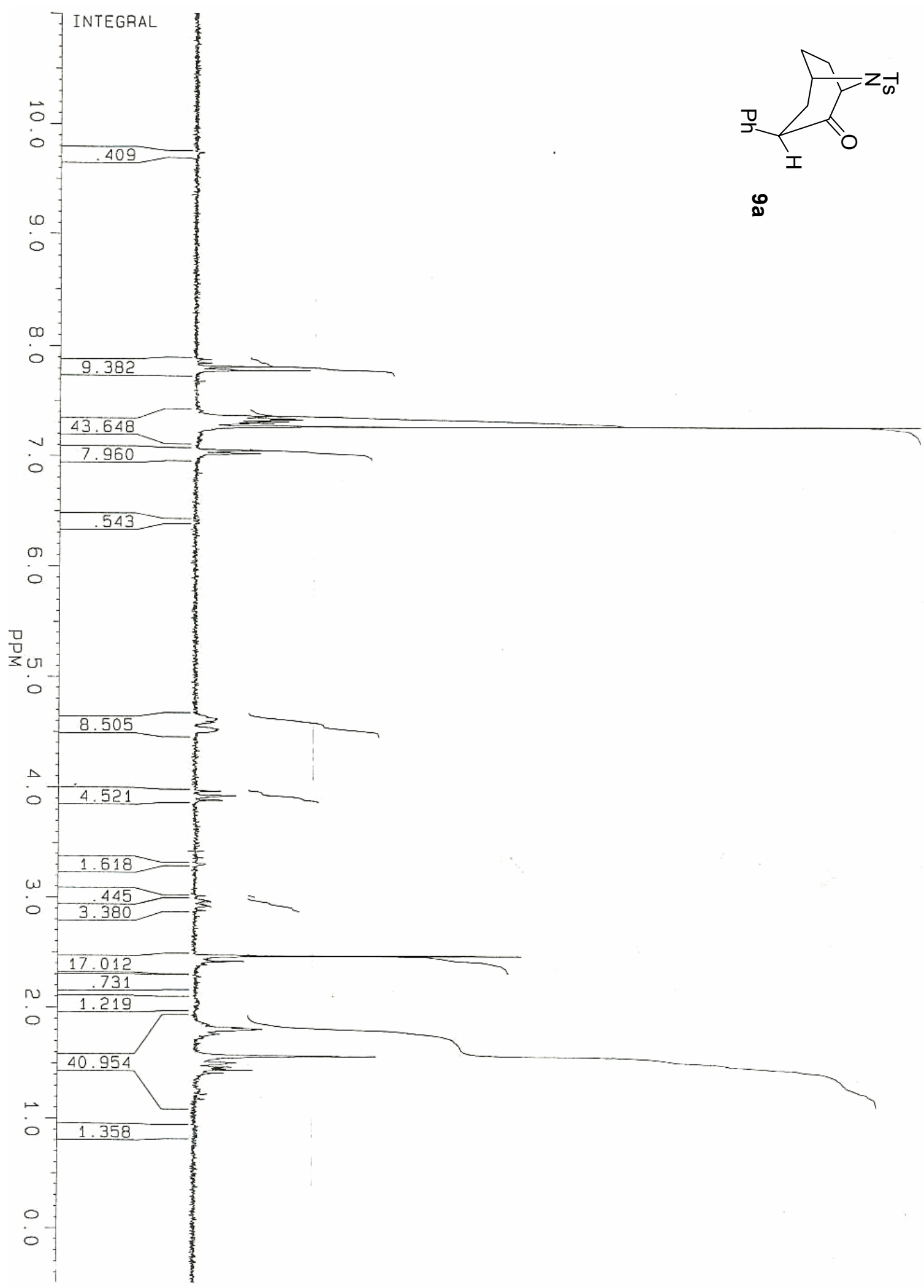



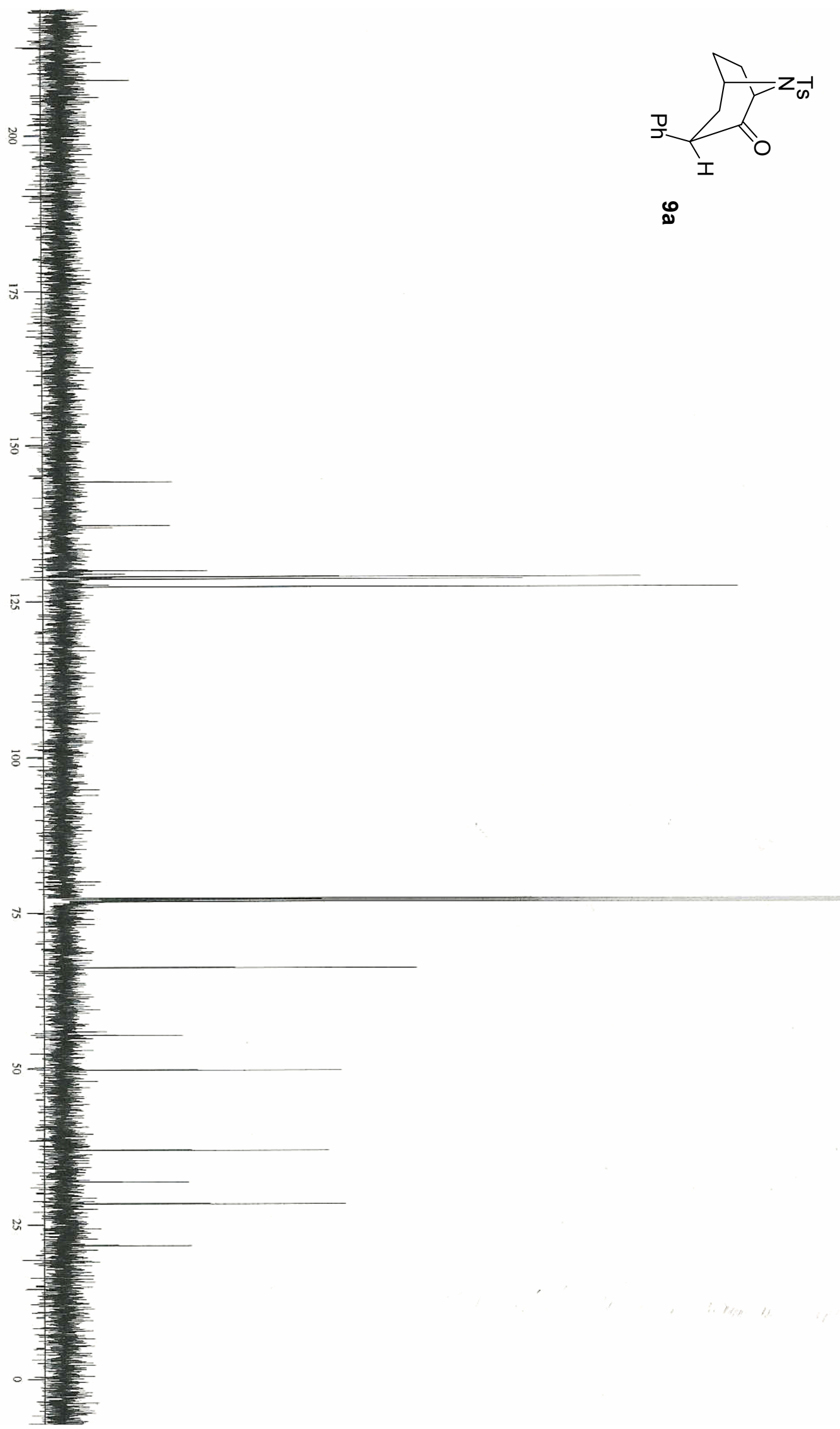


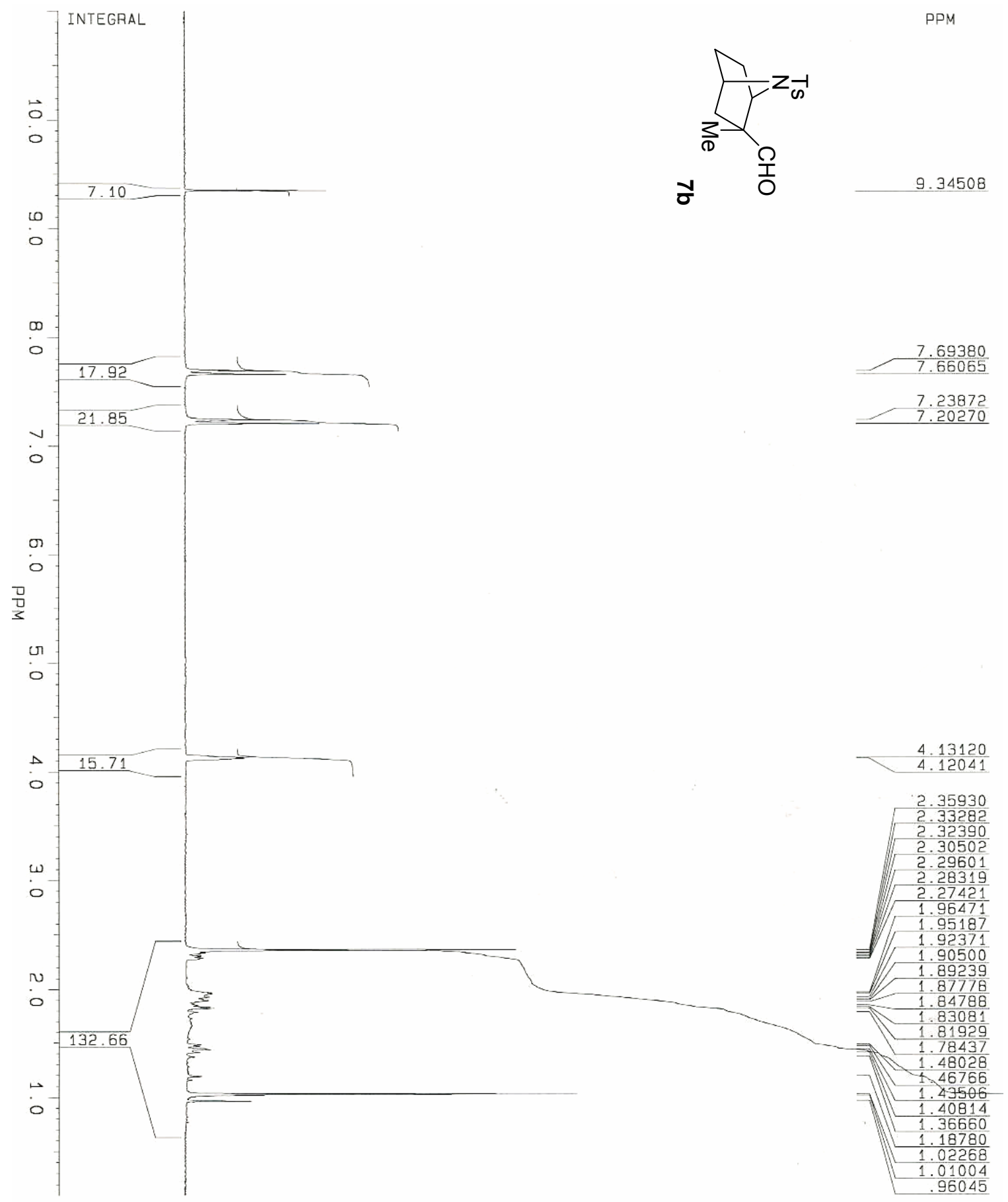




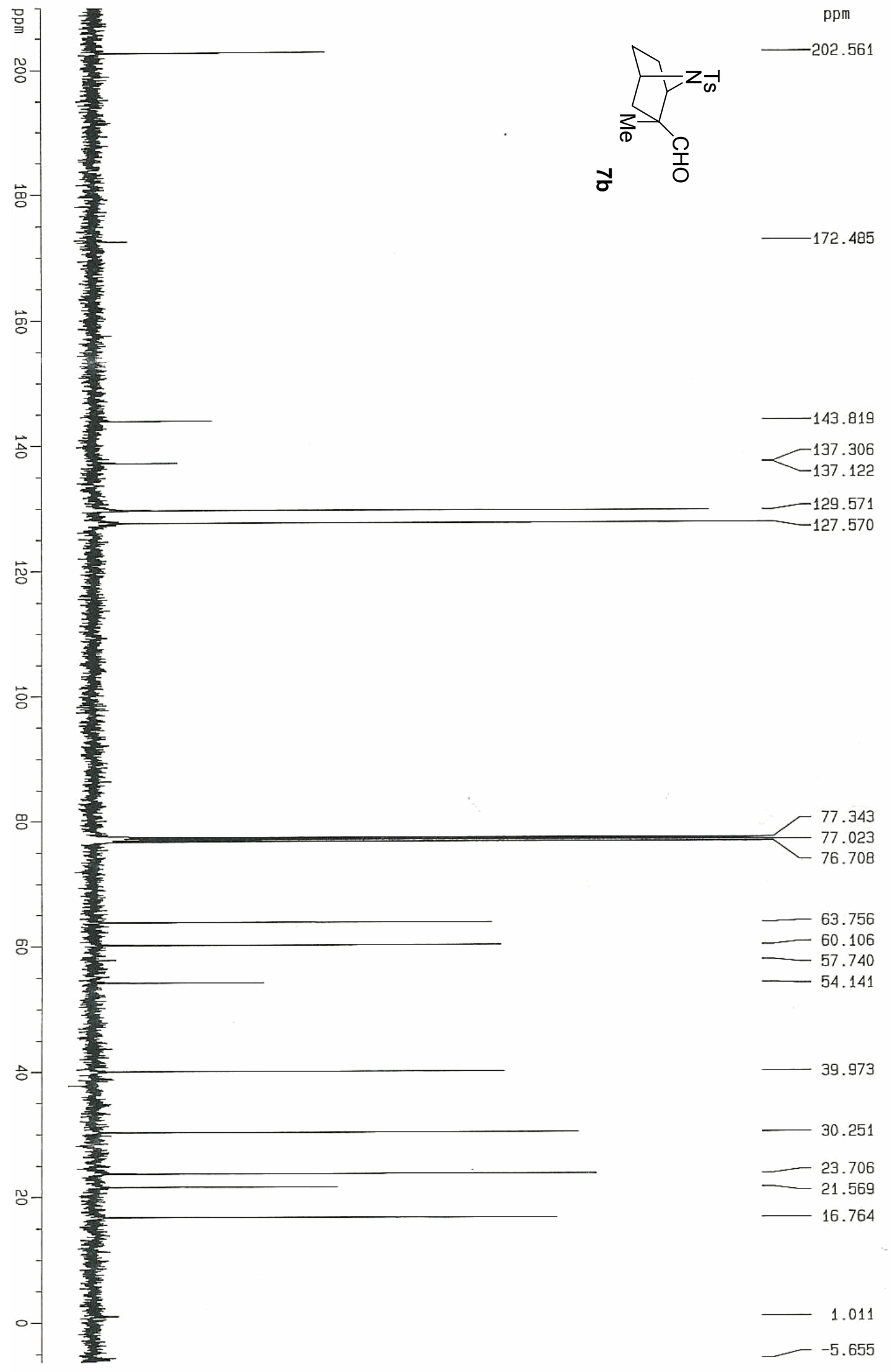




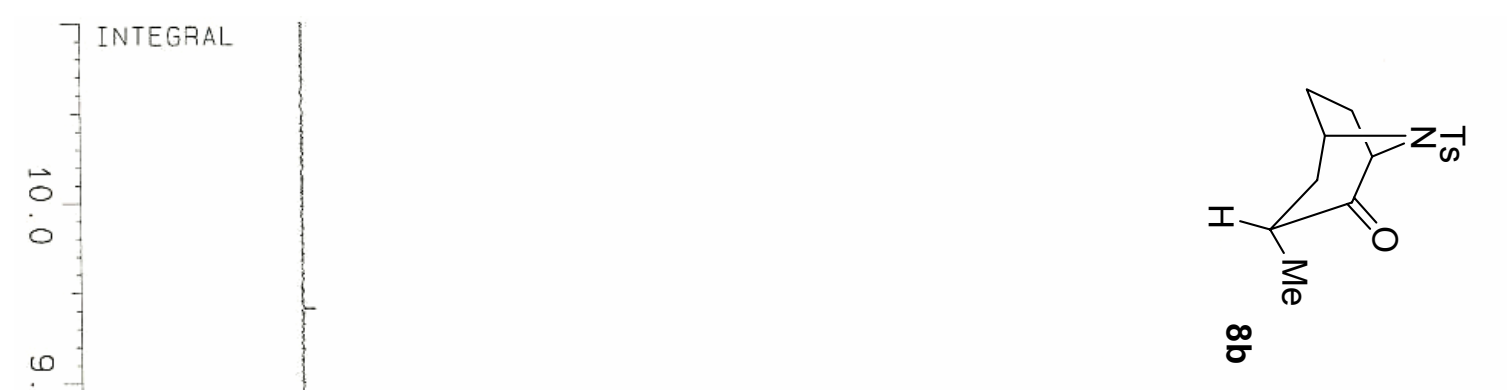




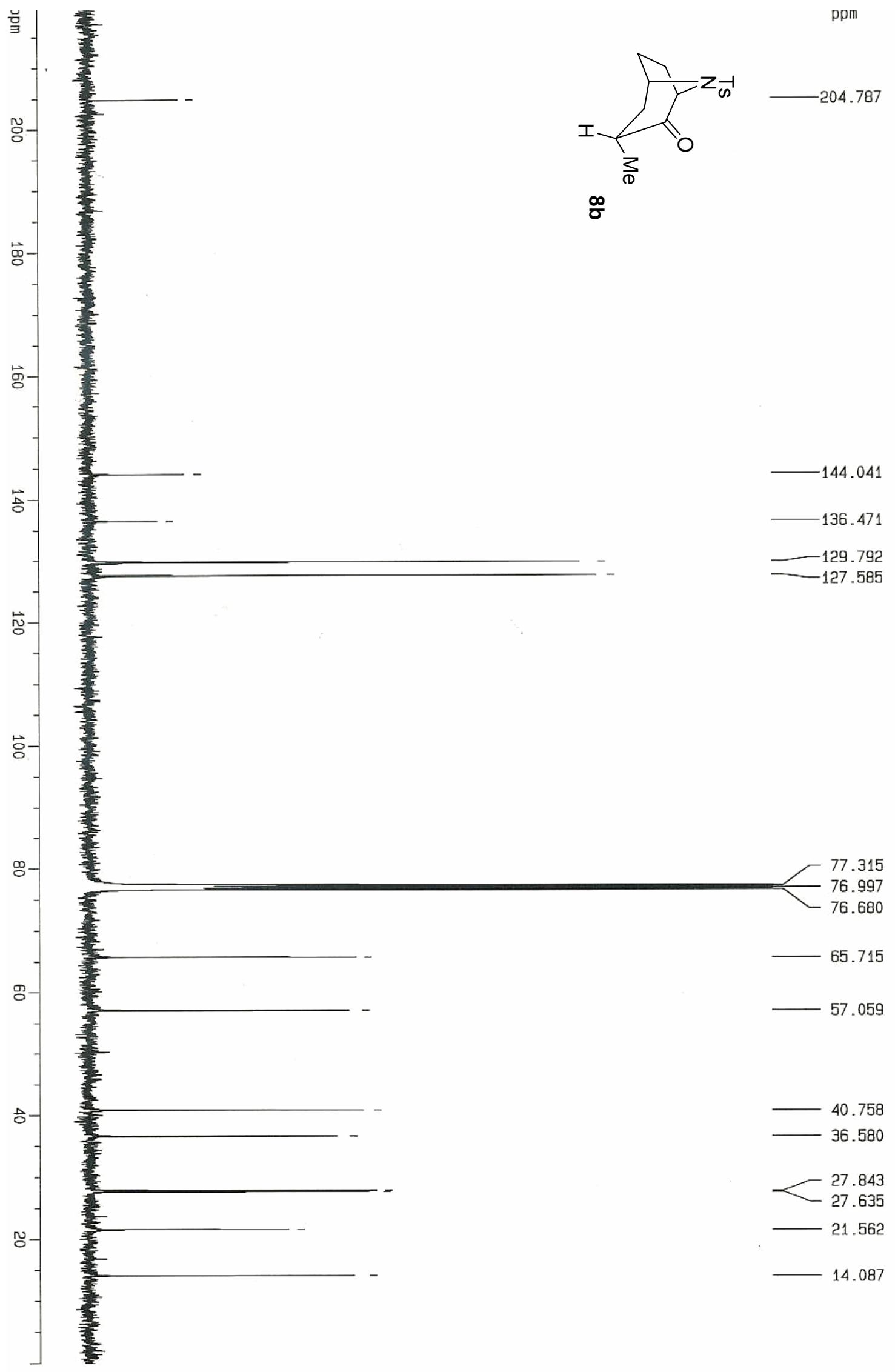




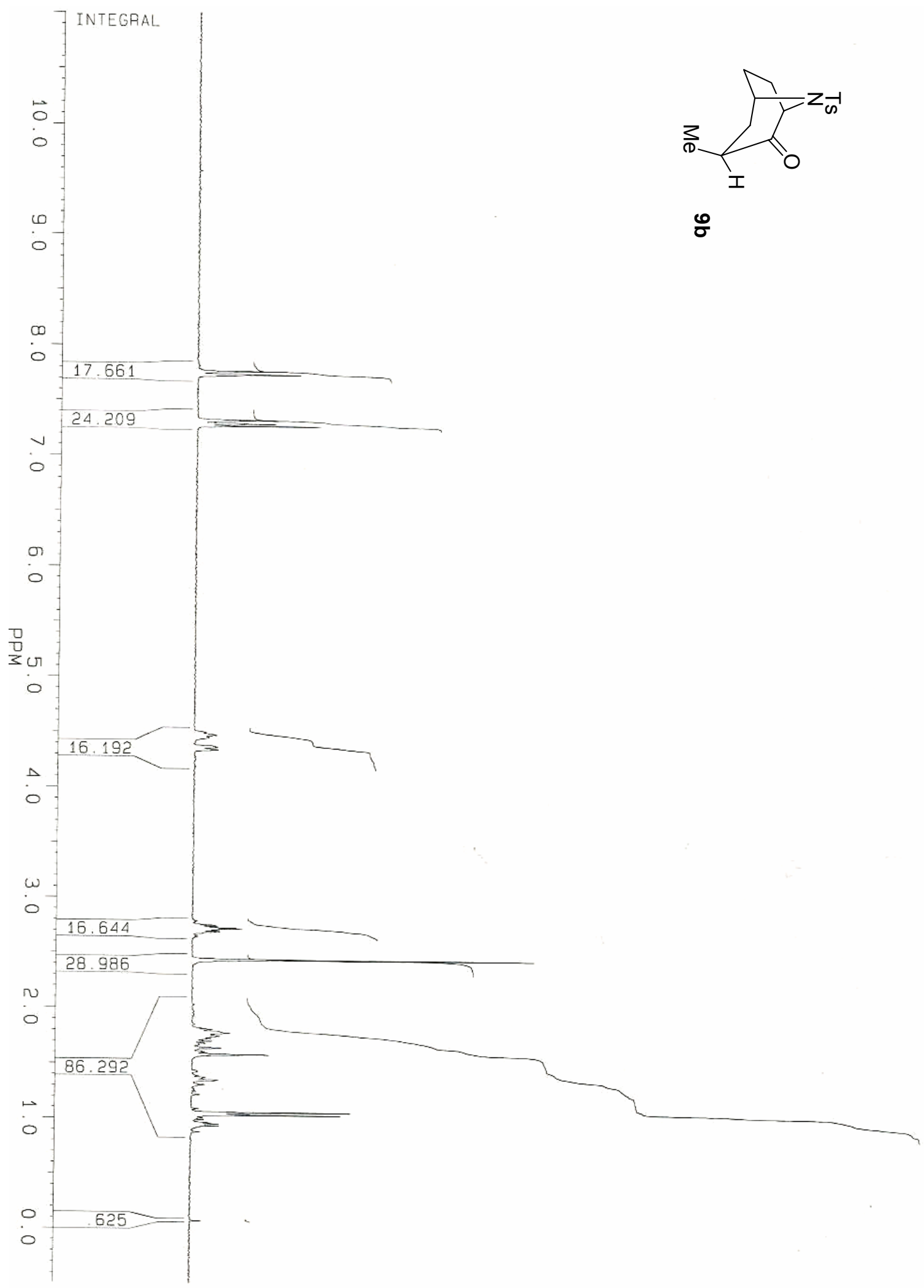




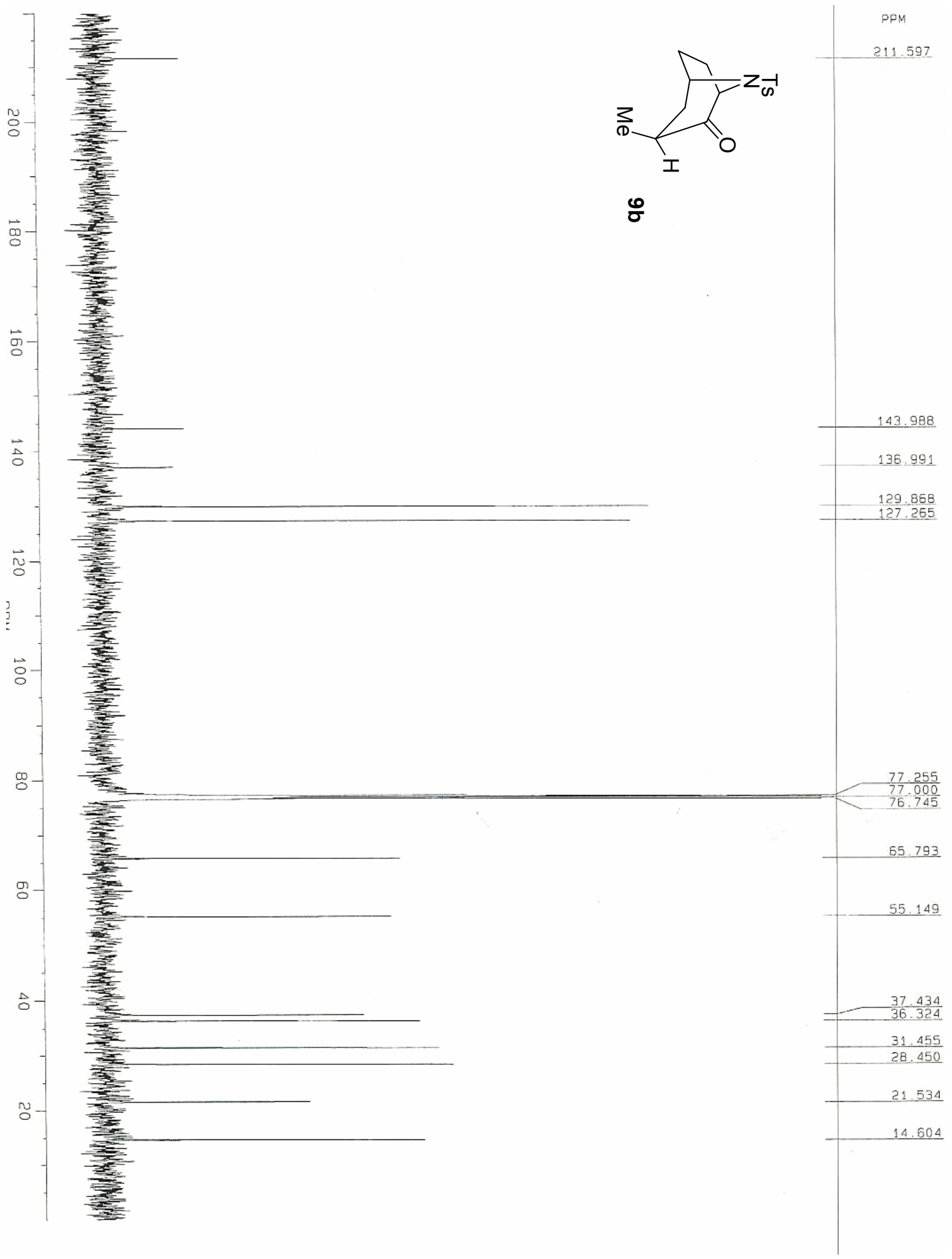




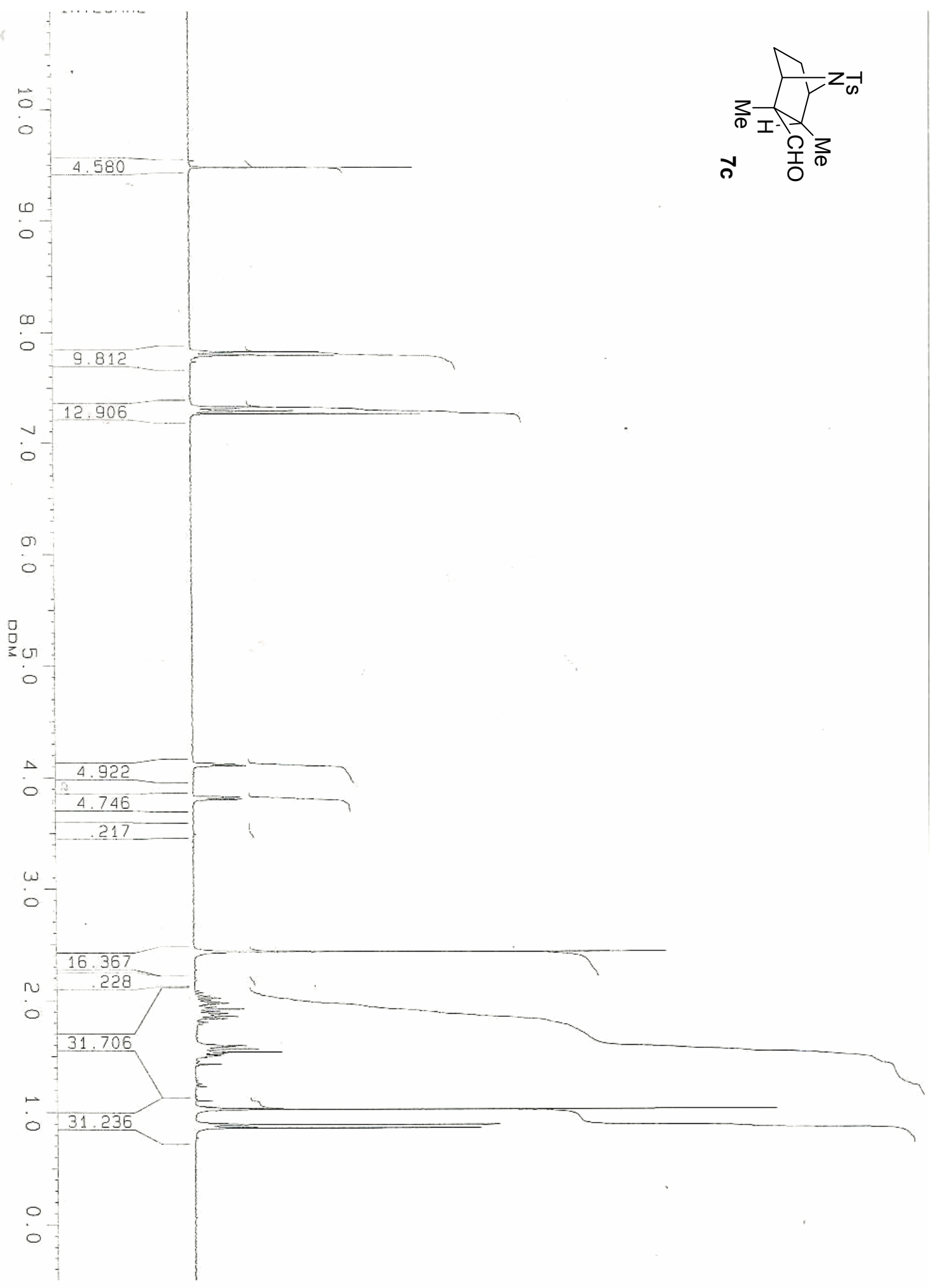




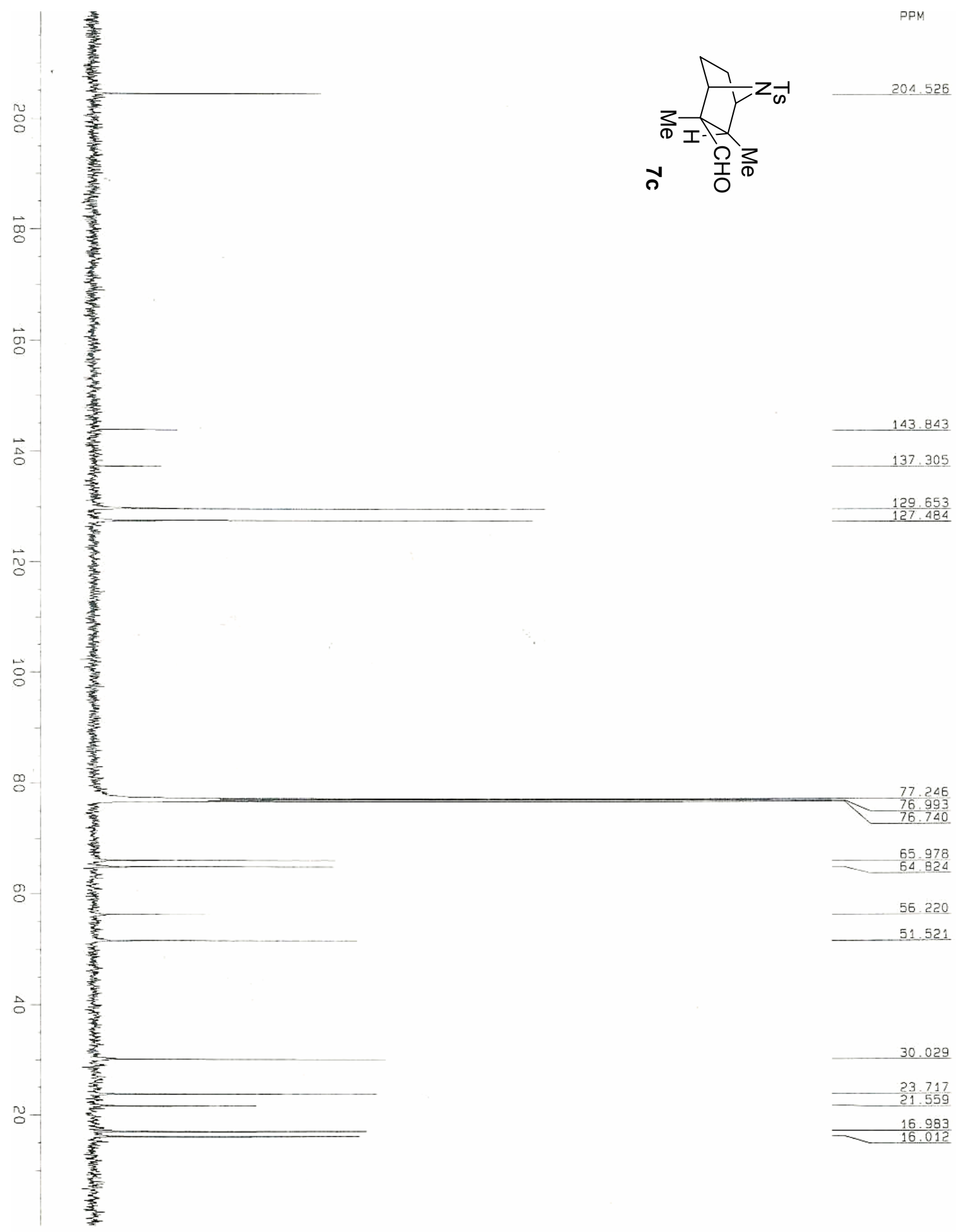




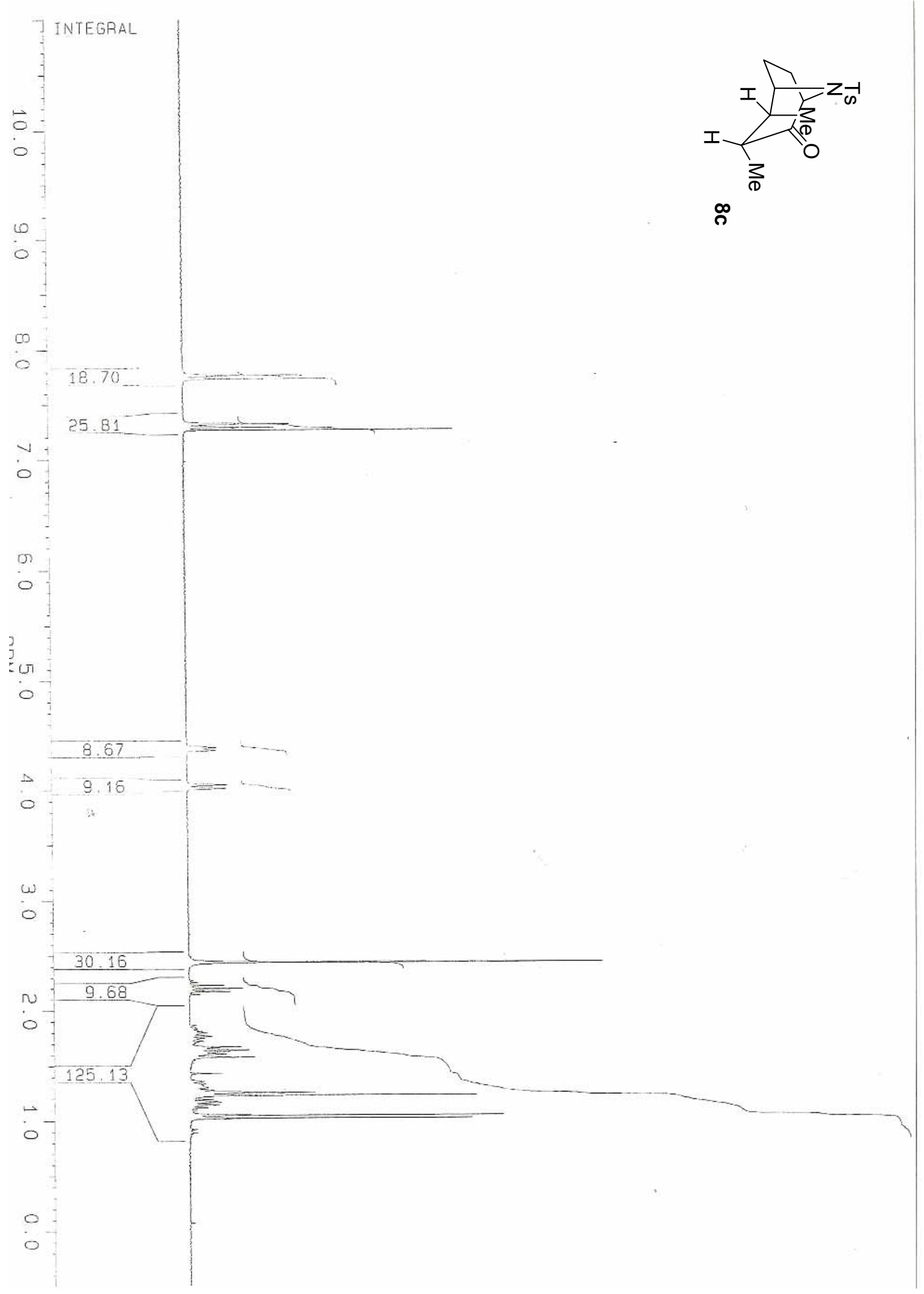




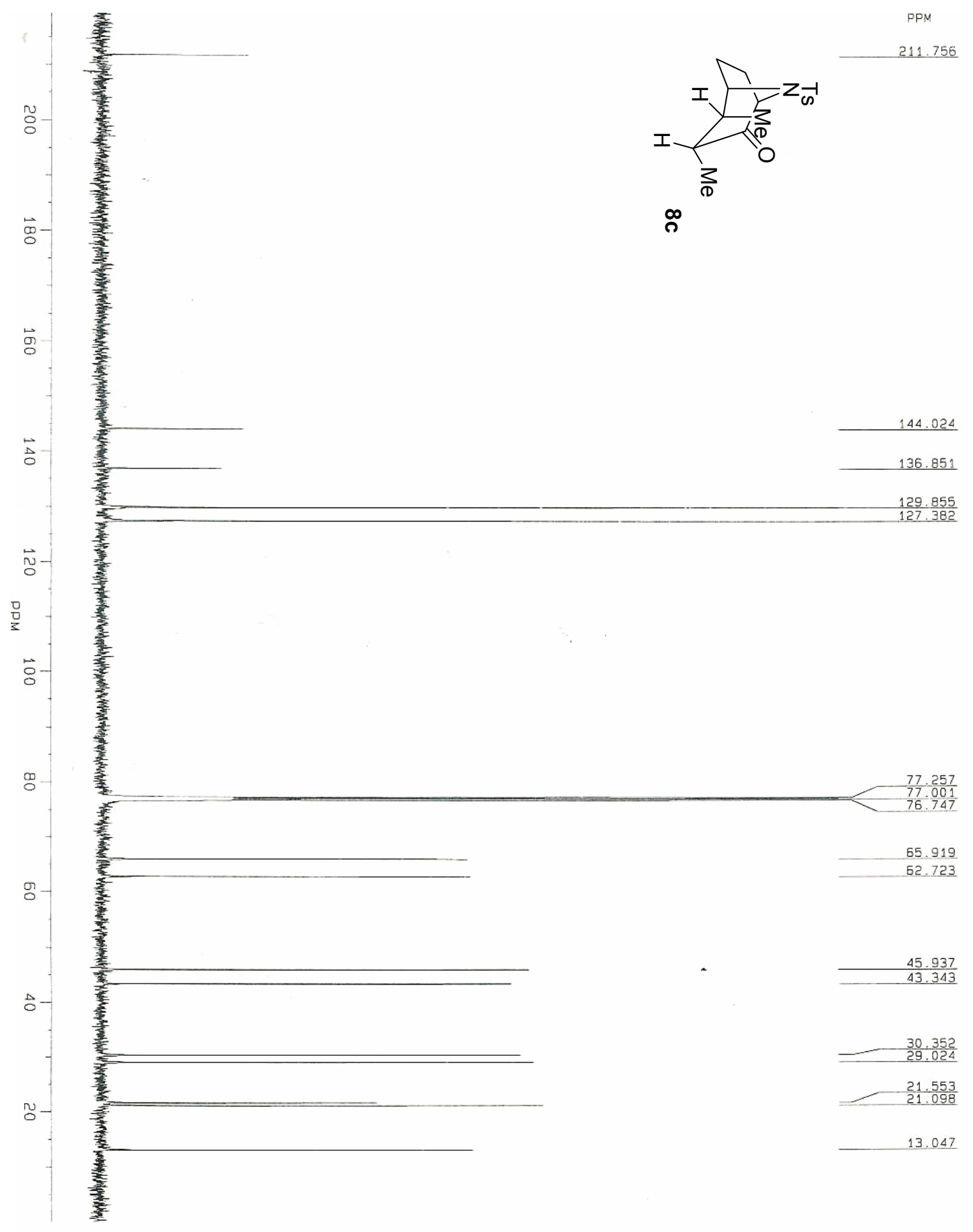




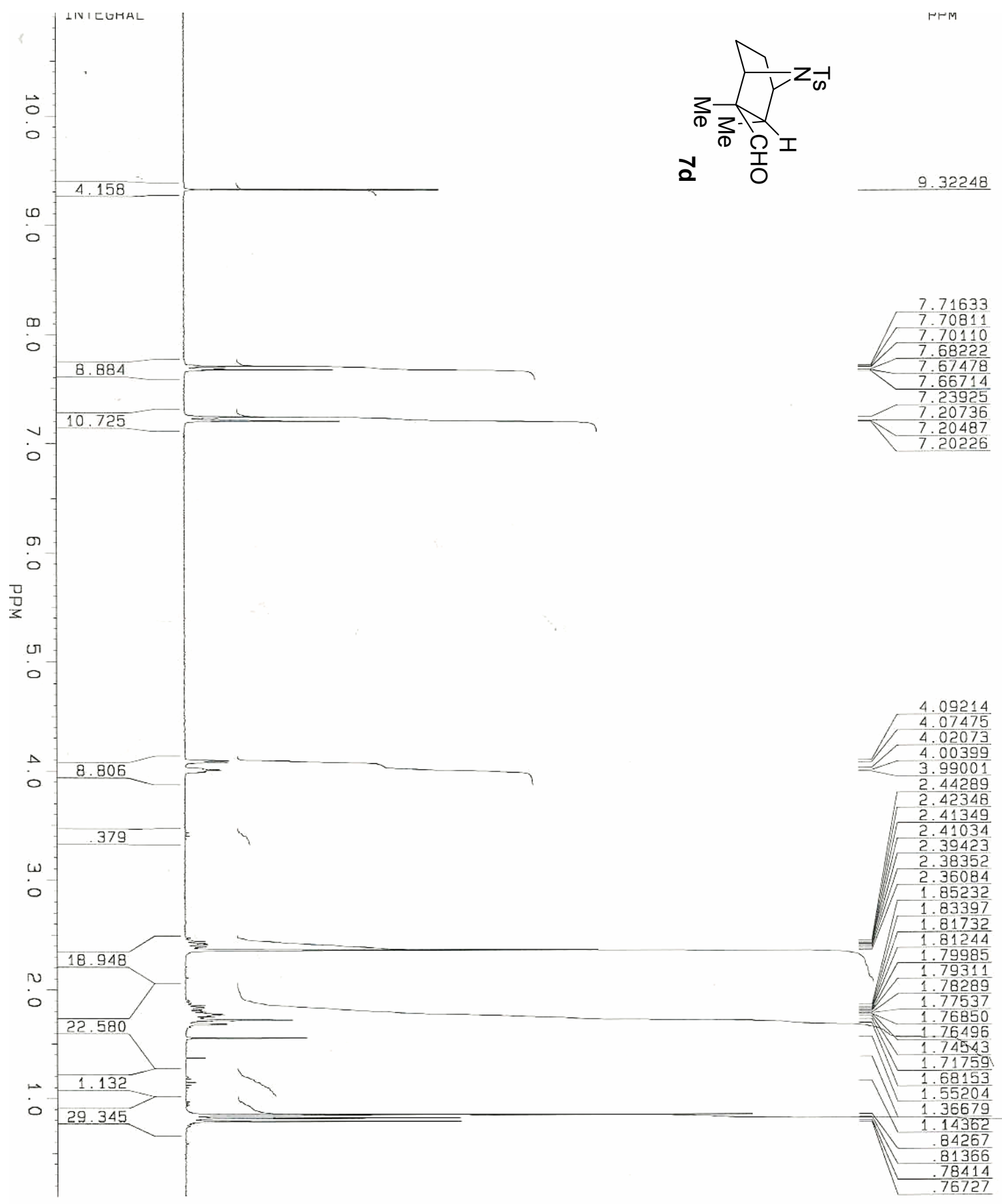




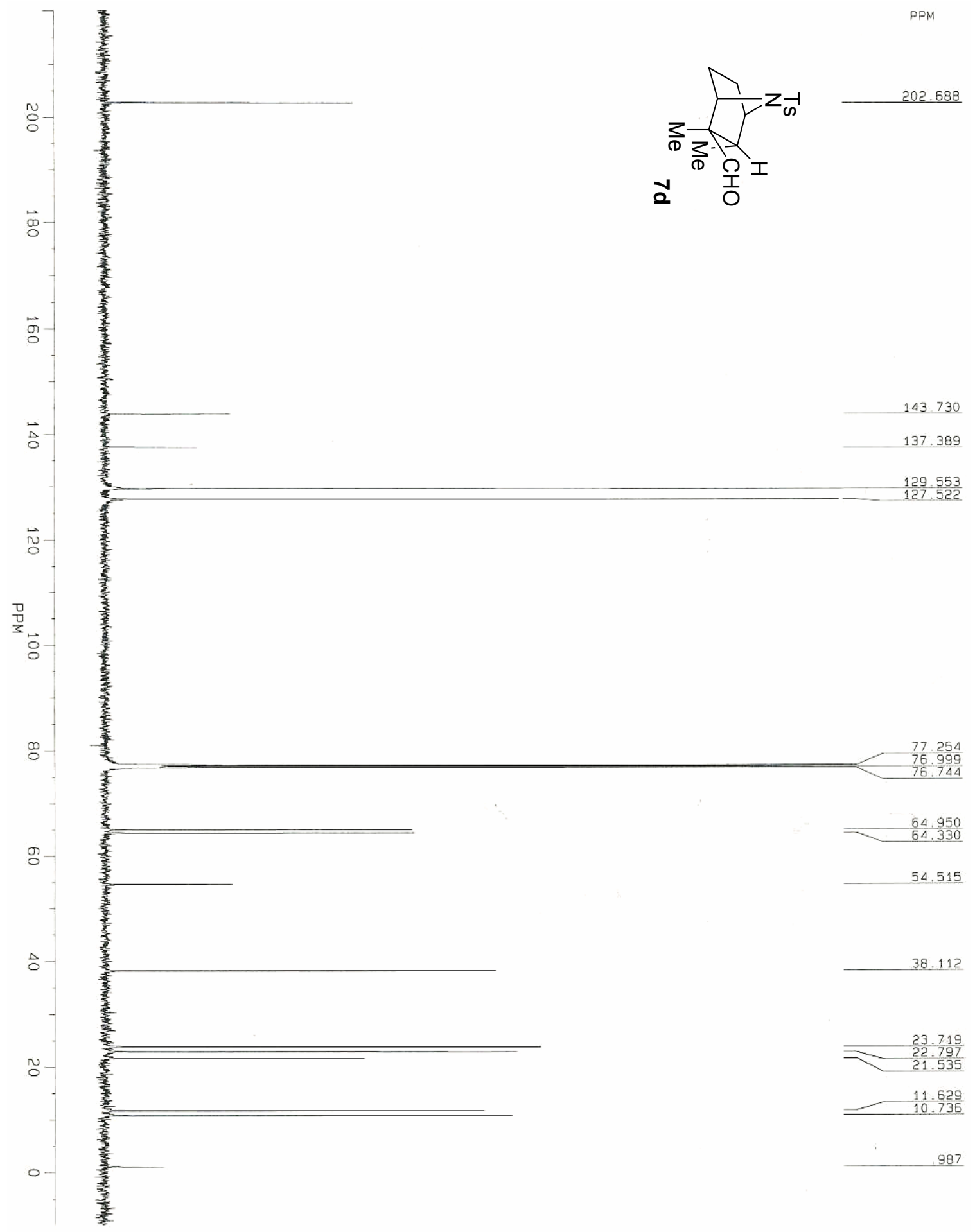




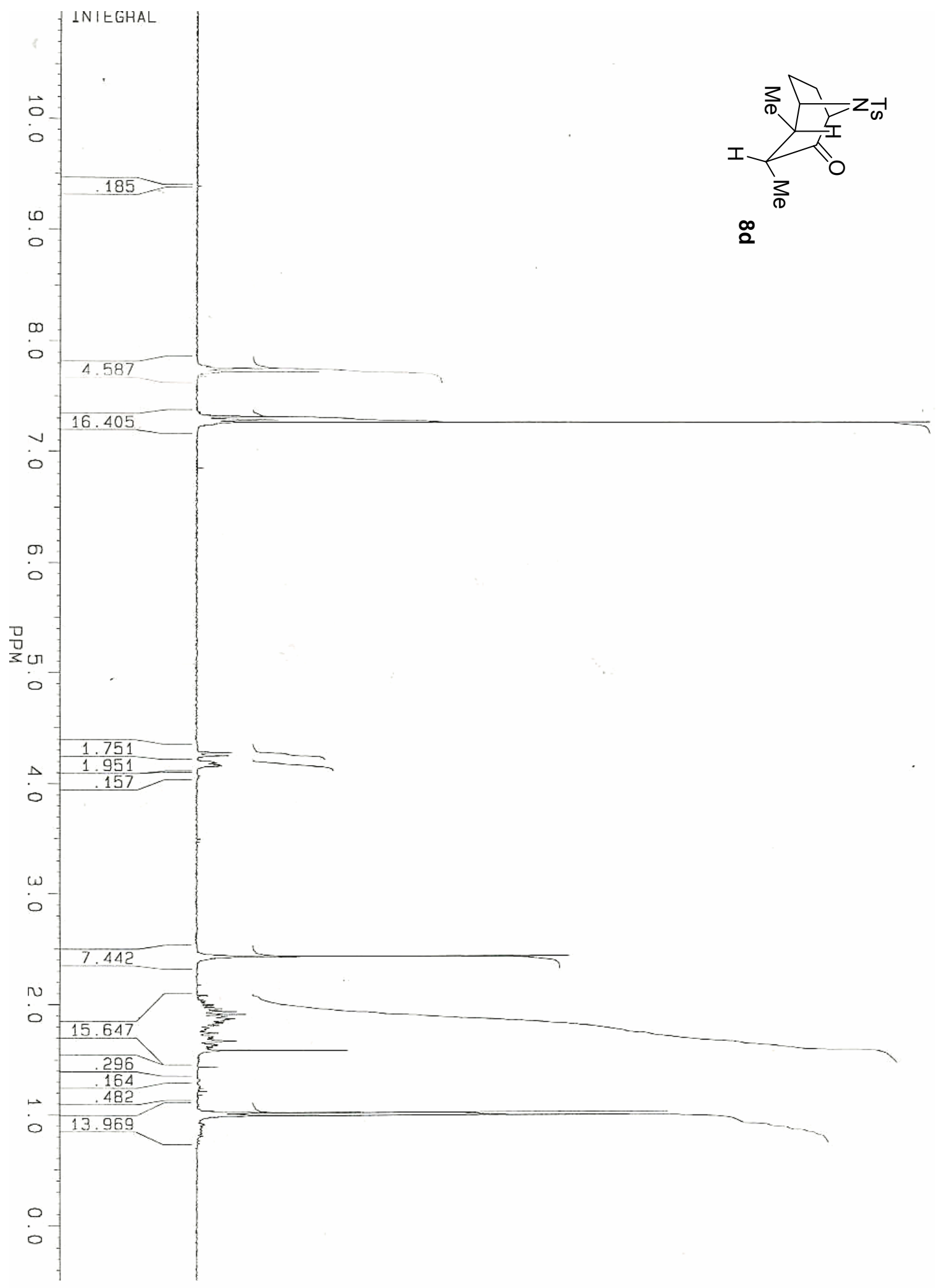




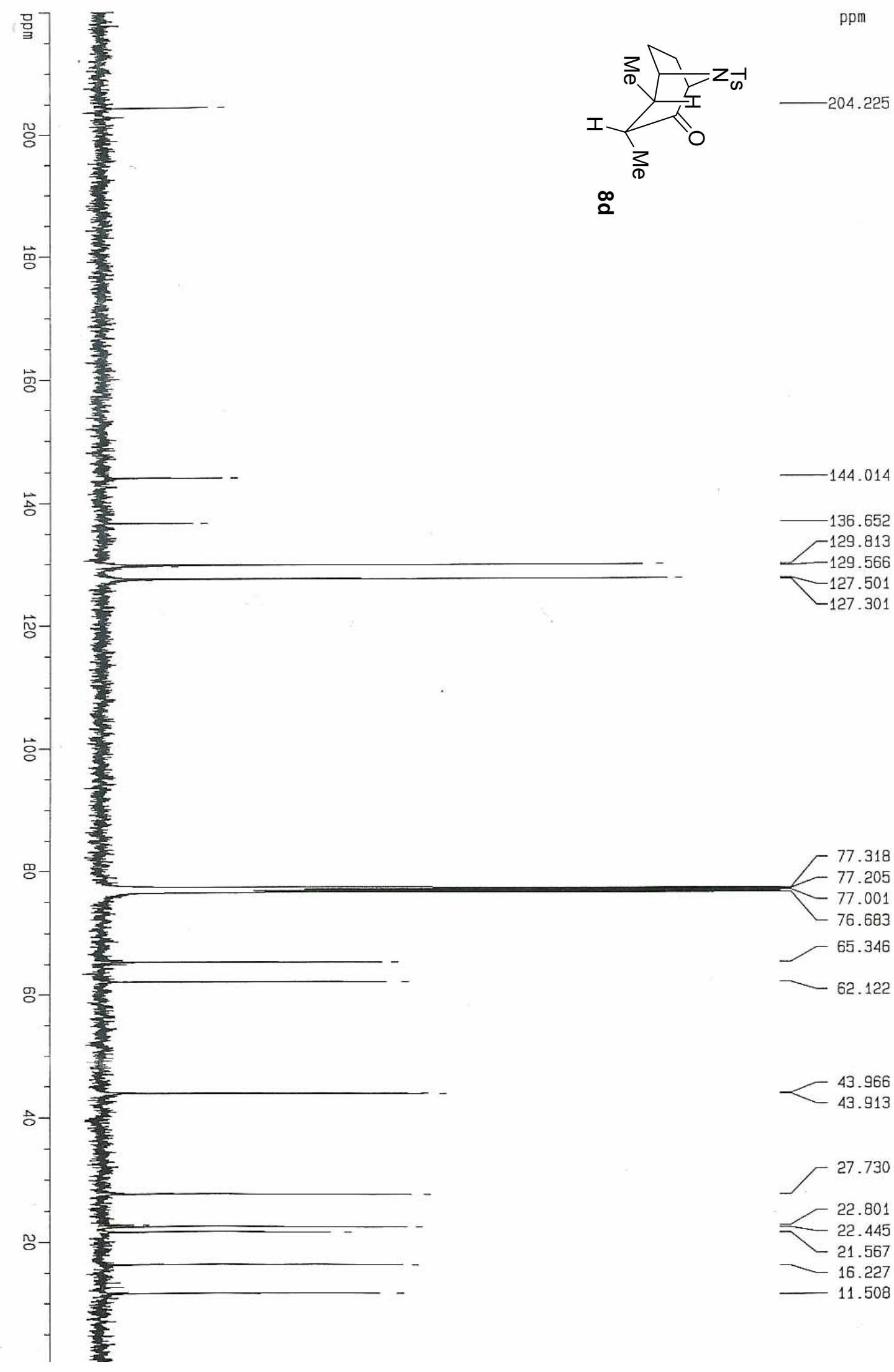




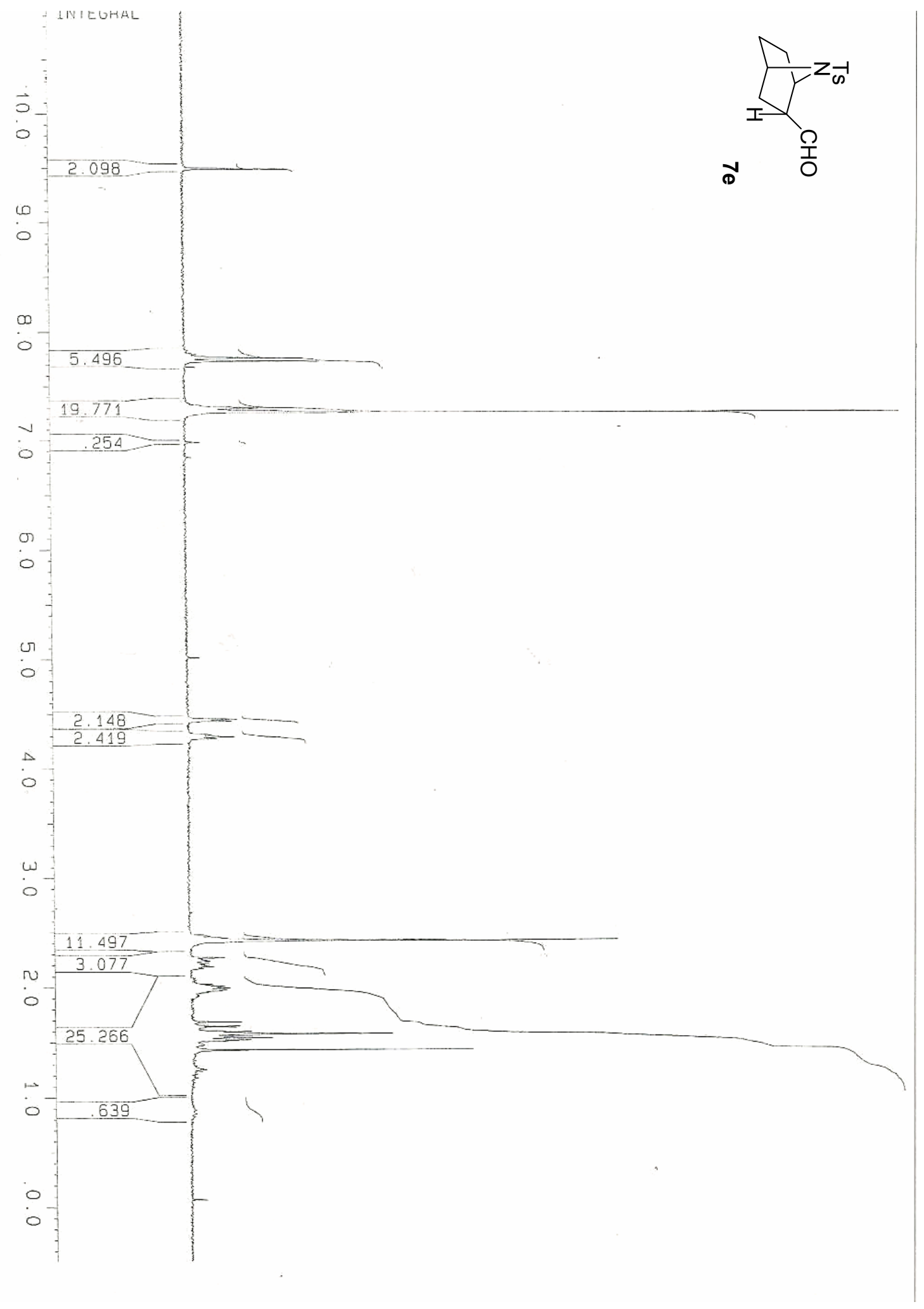




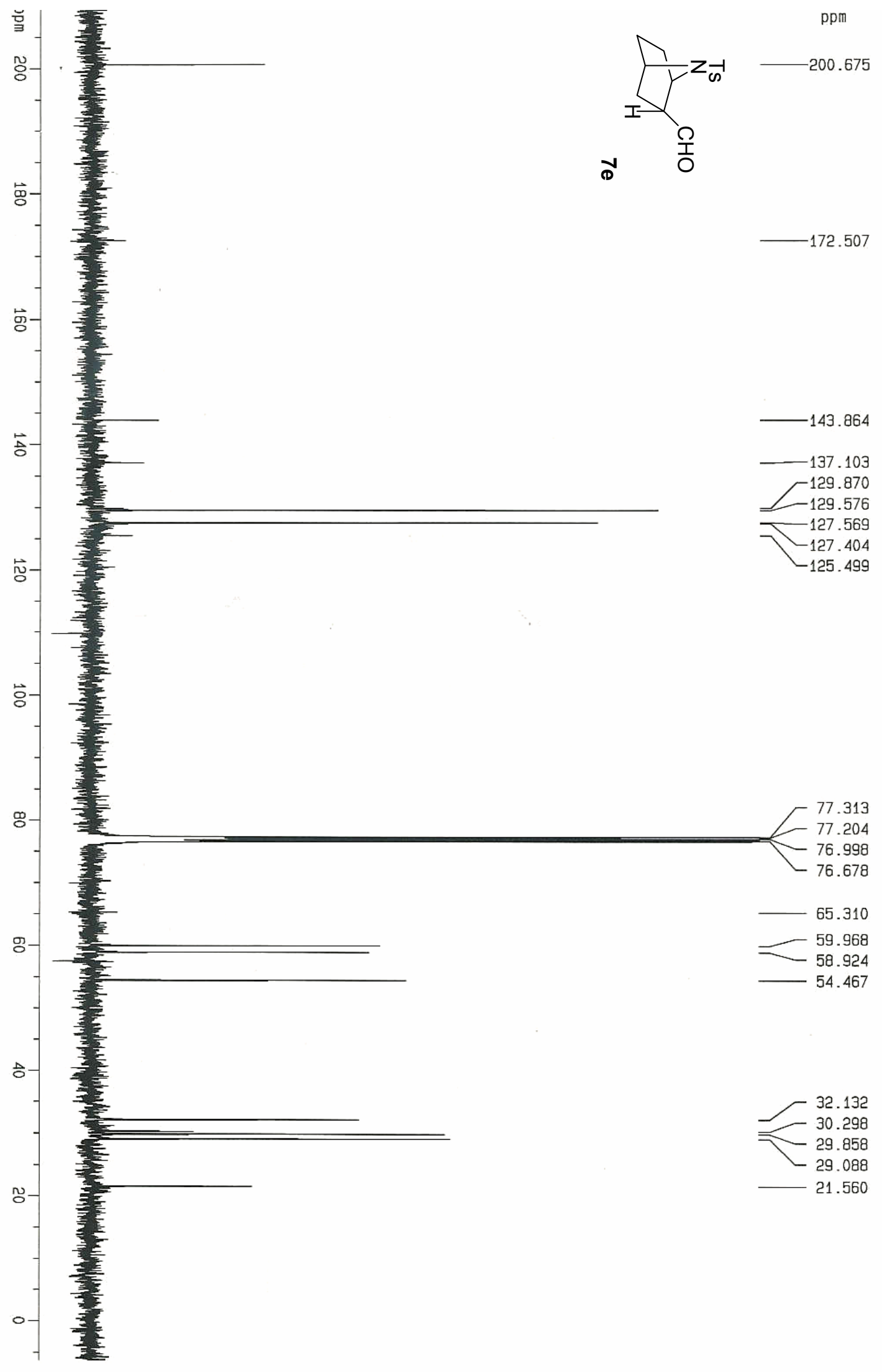




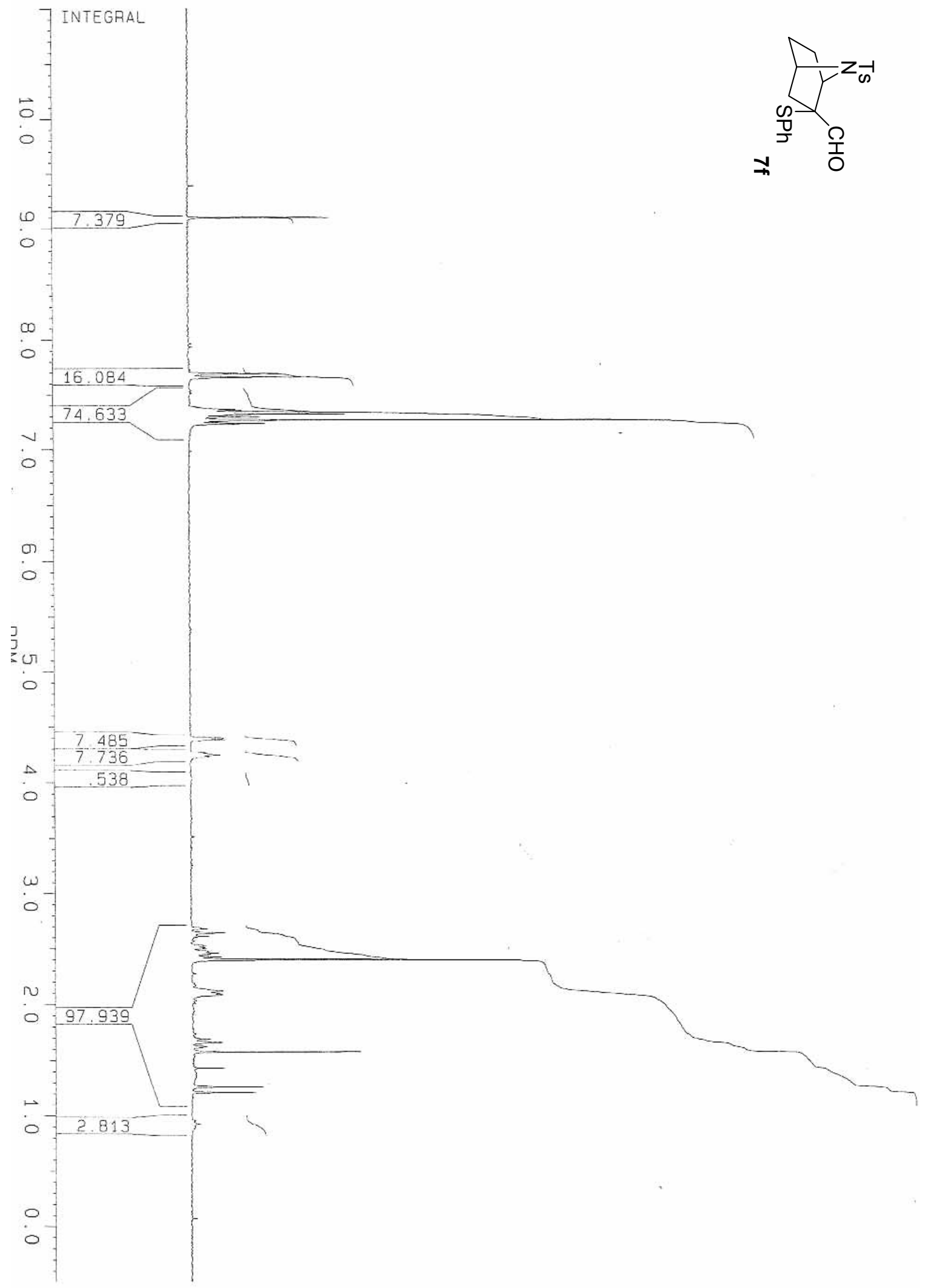




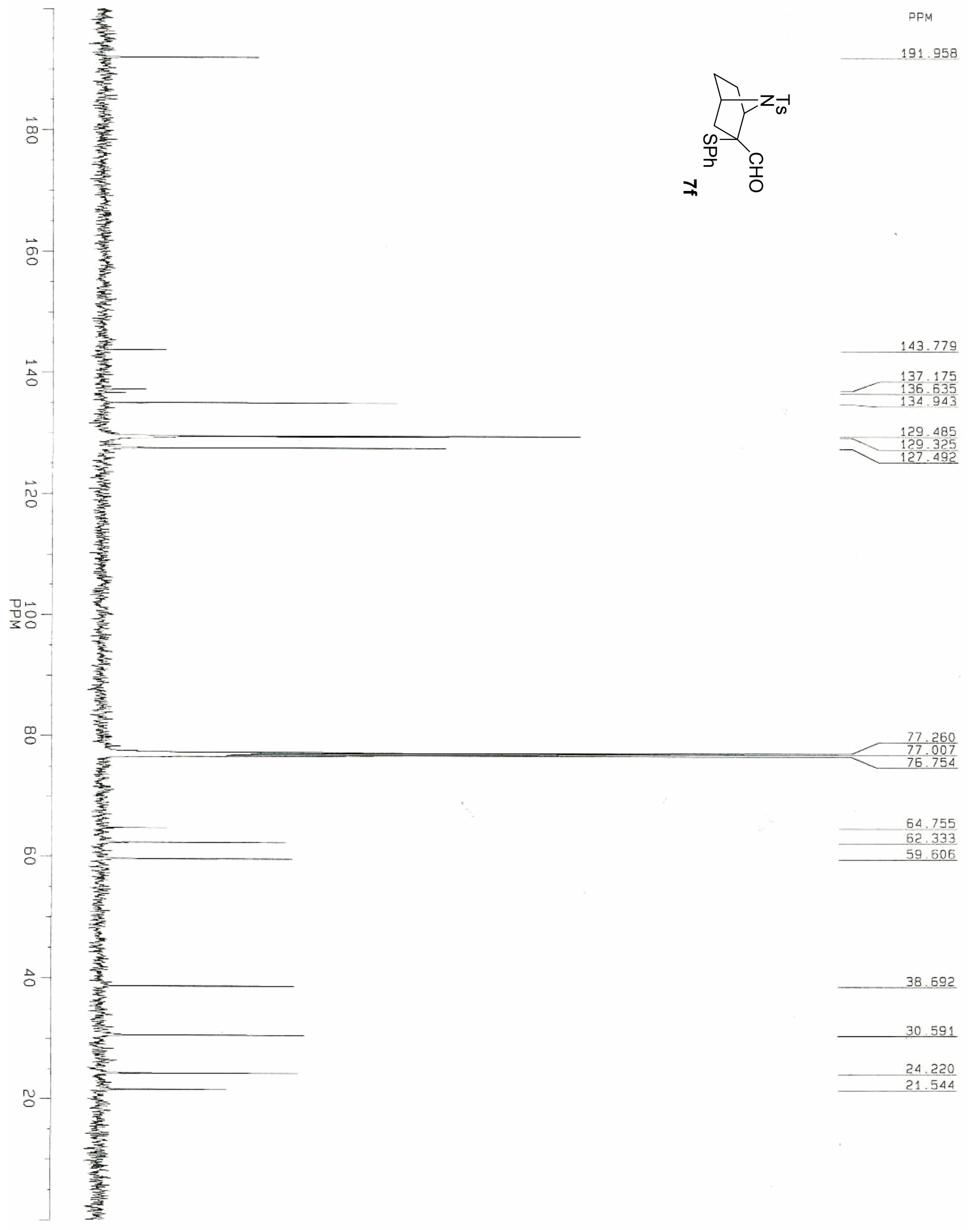




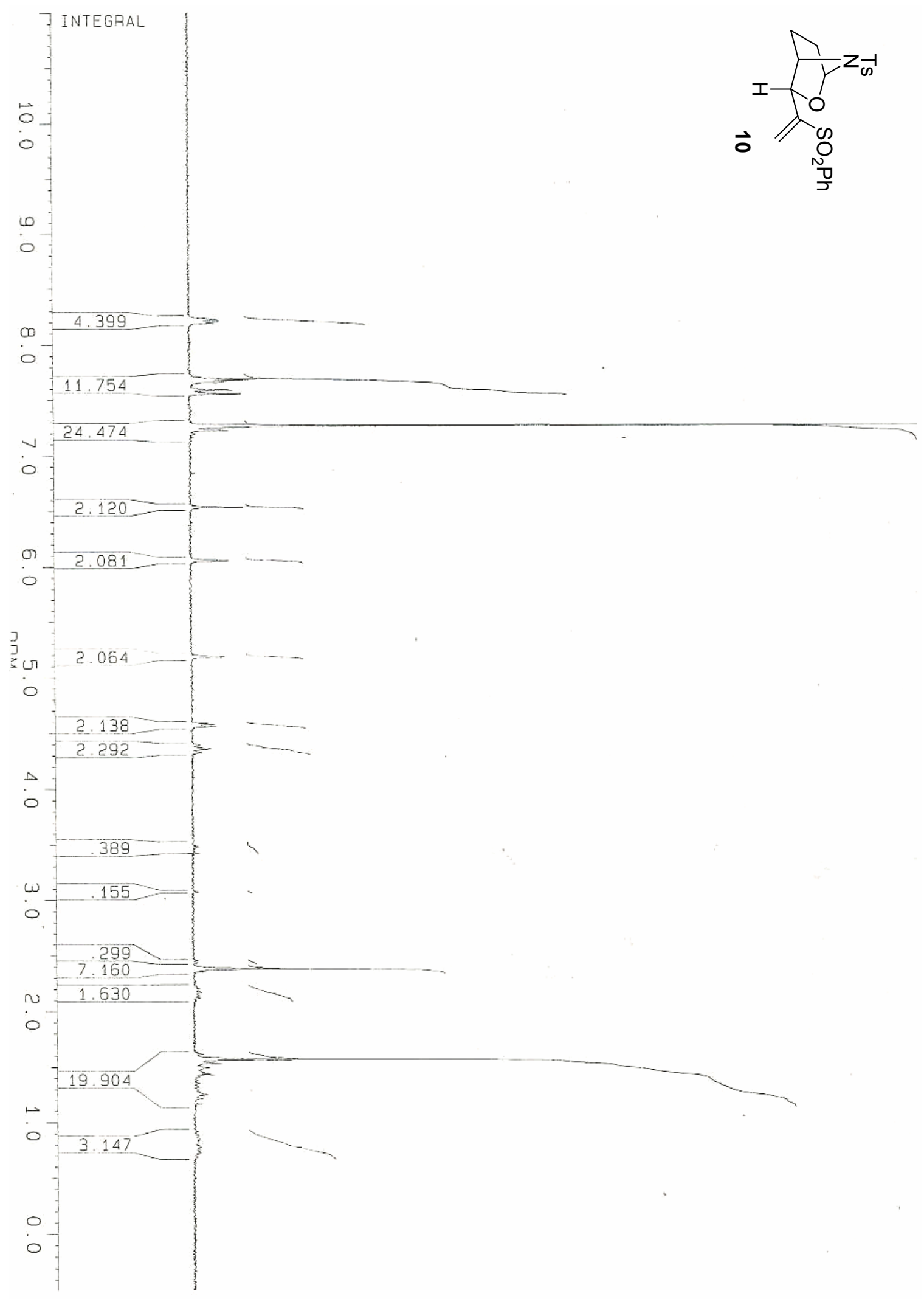




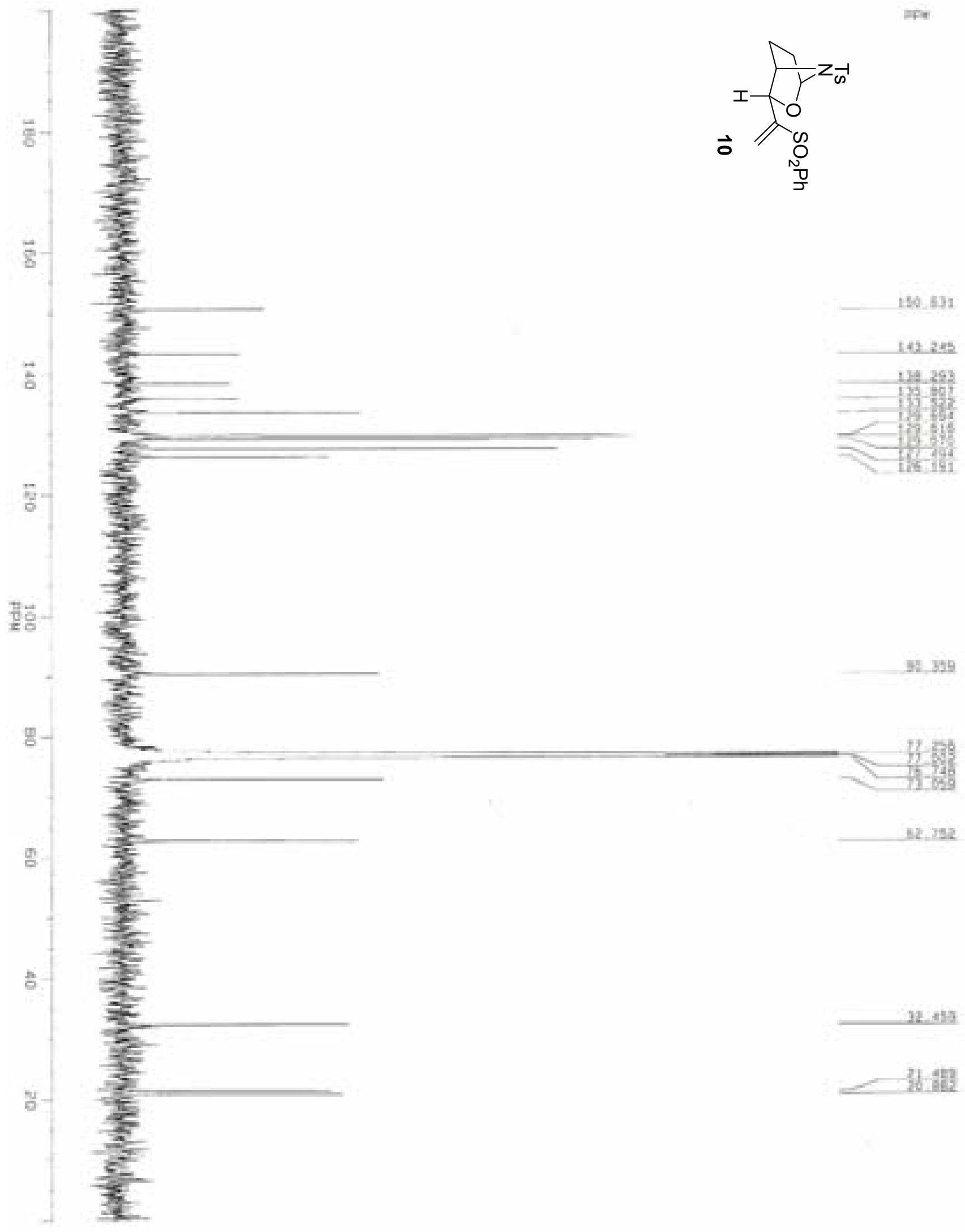




\section{$\underline{\text { Assignment of Stereochemical Configuration }}$}

\section{7-azabicyclo[2.2.1]heptanes}

The relative stereochemical configuration of products $7 \mathbf{a}$ and $\mathbf{7 b}$ was established on the basis of nOe studies (Figure 1).

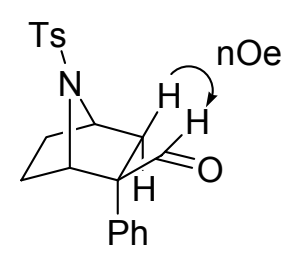

$7 a$

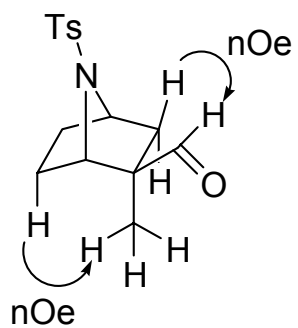

$7 b$

Figure 1

Much stereochemical information can be obtained from the observed magnitude of coupling constants around the [2.2.1] ring system (Figure 2).

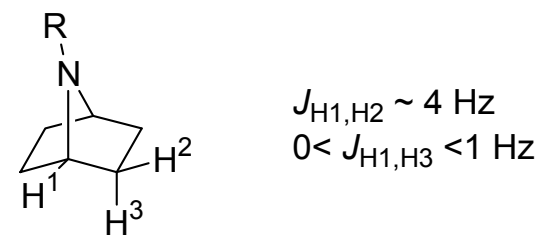

Figure 2

${ }^{3} J$ Coupling between bridgehead proton $\mathrm{H}^{1}$ and pseudo-equatorial $\mathrm{H}^{2}$ is readily observable. However, the dihedral angle between $\mathrm{H}^{1}$ and pseudo-axial $\mathrm{H}^{3}$ is close to $90^{\circ}$ and consequently ${ }^{3} J$ coupling is frequently negligible. This is the case for Epibatidine itself, ${ }^{1}$ and this effect has been observed with analogues, including one study where such findings were rationalised using in-silico molecular modelling. ${ }^{2}$

All compounds 7 have proton $\mathrm{nmr}$ spectra consistent with this model. Compounds $\mathbf{7 c}$ and 7e (Figure 3) give doublet signals for the emboldened protons, whilst in compound 7d, an isomer of 7c, the proton indicated gives a strongly triplet-like multiplet. The relative configuration of $\mathbf{7 e}$, and of the $-(\mathrm{CHMe})-$ methine centres in $\mathbf{7} \mathbf{c}$ and $\mathbf{7 d}$, are principally assigned on this basis. 


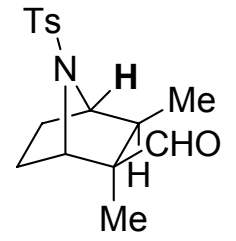

7c

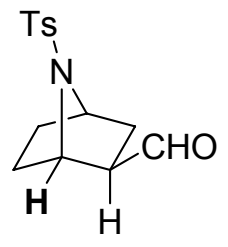

$7 e$

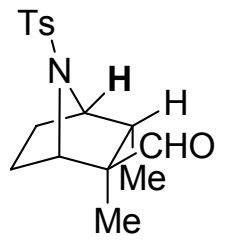

7d

Figure 3

Furthermore, nOe experiments (Figure 4) on 7c and 7d showed interactions establishing the exo orientation of their aldehyde groups.

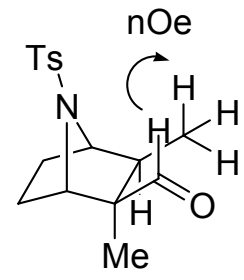

7c

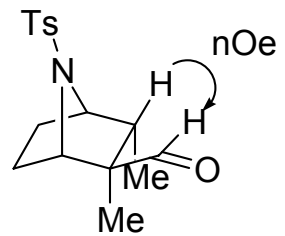

7d

Figure 4

\section{[3.2.1] Tropanes}

The relative configuration of major products $8 \mathbf{a}$ and $\mathbf{8 b}$ was established on the basis of nOe studies (Figure 5).
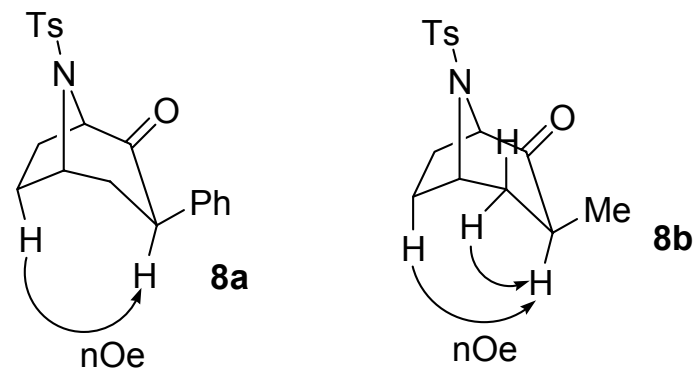

Figure 5

As is normally the case, ${ }^{3}$ an equatorial proton at position C-3 in compounds 9 gives a proton $\mathrm{nmr}$ signal at markedly lower field than an axial proton in isomeric tropanes $\mathbf{8}$ 
(Table 1). In this regard, the spectrum of trace product $9 \mathbf{c}$ is useful in assigning $\mathrm{C}-3$ configuration to 8c (Figure 6).
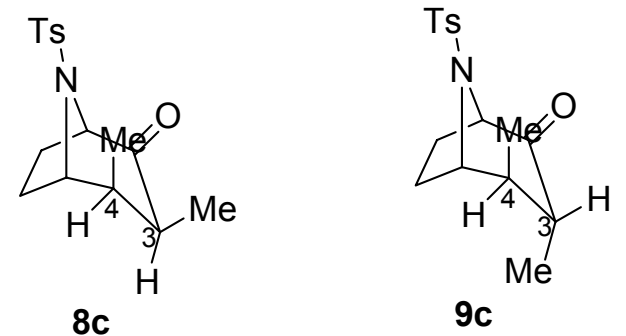

Figure 6

Table 1

\begin{tabular}{|l|l|l|l|}
\hline \multicolumn{4}{|c|}{ C-3 methine proton chemical shift $\delta(\mathrm{ppm})$} \\
\hline $\mathbf{8 a}$ & 3.59 & $\mathbf{9 a}$ & 3.92 \\
\hline $\mathbf{8 b}$ & $2.51-2.34(\mathrm{~m})$ & $\mathbf{9 b}$ & $2.80-2.62(\mathrm{~m})$ \\
\hline $\mathbf{8 c}$ & 2.20 & $\mathbf{9 c}$ & 2.58 \\
\hline
\end{tabular}

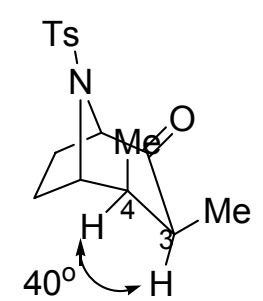

$8 c$

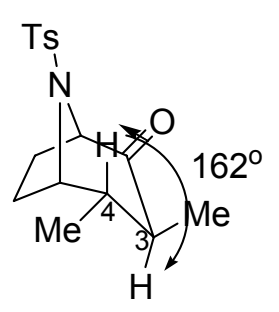

8d

Figure 7. Predicted dihedral angles

Dihedral angles between the C-3 and C-4 methine hydrogens of $8 \mathbf{c}$ and $8 \mathbf{d}$ were predicted using in-silico molecular modelling (Spartan ${ }^{\circledR}$ software, Figure 7). The predicted angle for $8 \mathbf{c}$ is $40^{\circ}$, which is consistent with the observed ${ }^{3} J$ coupling of $6.8 \mathrm{~Hz}$. The magnitude of this coupling constant between C-3 and C-4 hydrogens was similar for $9 \mathbf{c}(7.0 \mathrm{~Hz})$. However, the dihedral angle for $\mathbf{8 d}$ is predicted to be $162^{\circ}$. Karplus curve analysis, assuming a typical ${ }^{3} J$ coupling of $12 \mathrm{~Hz}$ for antiperiplanar $\left(180^{\circ}\right)$ alignment in a rigid cyclohexane, gives a predicted value of $J \approx 10-11 \mathrm{~Hz}$. Selective decoupling of the two ring system methyl groups of $\mathbf{8 d}$ in its $500 \mathrm{MHz}$ proton $\mathrm{nmr}$ spectrum permitted an experimental measurement of $J=10.8 \mathrm{~Hz}$. This is only consistent with a pseudotransdiaxial coupling. 
${ }^{1}$ Spande, T. F.; Garraffo, H. M.; Edwards, M. W.; Yeh, H. J. C.; Pannell, L.; Daly, J. W. J. Am. Chem. Soc. 1992, 114, 3475-3478.

${ }^{2}$ Clayton, S. C.; Regan, A. C. Tetrahedron Lett. 1993, 34, 7493-7496.

${ }^{3}$ Williams, D. H.; Fleming, I. Spectroscopic Methods In Organic Chemistry; McGraw-Hill, 1995, p. 129. 